\title{
A semi-implicit approach for fluid-structure interaction based on an algebraic fractional step method
}

\author{
Annalisa Quaini ${ }^{\dagger}$, Alfio Quarteroni ${ }^{\dagger *}$
}

23rd October 2006

\author{
${ }^{\dagger}$ Chair of Modelling and Scientific Computing (CMCS), \\ Institute of Analysis and Scientific Computing (IACS), \\ École Polytechnique Fédérale de Lausanne, \\ CH-1015, Lausanne, Switzerland. \\ annalisa.quaini@epfl.ch \\ * MOX-Modellistica e Calcolo Scientifico \\ Dipartimento di Matematica "F. Brioschi" \\ Politecnico di Milano \\ via Bonardi 9, 20133 Milano, Italy \\ alfio.quarteroni@epfl.ch
}

Keywords: Fluid-structure interaction; semi-implicit coupling; algebraic fractional step; Yosida method; added-mass effect; blood flows.

AMS Subject Classification: 74F10, 65N30, 76D05

\begin{abstract}
We address the numerical simulation of fluid-structure interaction problems dealing with an incompressible fluid whose density is close to the structure density. We propose a semi-implicit coupling scheme based on an algebraic fractional-step method. The basic idea of a semi-implicit scheme consists in coupling implicitly the added-mass effect, while the other terms (dissipation, convection and geometrical non-linearities) are treated explicitly. Thanks to this kind of explicit-implicit splitting, computational costs can be reduced (in comparison to fully implicit coupling algorithms) and the scheme remains stable for a wide range of discretization parameters. In this paper we propose to derive this kind of splitting from the algebraic formulation of the coupled fluid-structure problem (after finite-element space discretization). This approach extends for the first time to fluid-structure problems the algebraic fractional step methodology that was previously advocated to treat the pure fluid problem in a fixed domain. More particularly, for the specific semi-implicit method presented in this report we adapt the Yosida scheme to the case of a coupled fluid-structure problem.
\end{abstract}


This scheme relies on an approximate $L U$ block factorization of the matrix obtained after the discretization in time and space of the fluid-structure system.

We analyze the numerical performances of this scheme on 2D fluid-structure simulations performed with a simple 1D structure model.

\section{Introduction}

We consider a time dependent computational domain $\Omega(t)$ such that $\bar{\Omega}(t)=$ $\bar{\Omega}^{f}(t) \cup \bar{\Omega}^{s}(t)$ in $\mathbb{R}^{d}(d=2$ or 3 ), consisting, at any time $t>0$, of a deformable structure $\Omega^{s}(t)$ surrounding a moving volume $\Omega^{f}(t)$ filled by fluid under motion (see Fig. 1). The initial configuration at time $t=0$ will be indicated with the "hat" overscript, that is $\hat{\Omega}=\Omega(0)$ and $\hat{\Omega}^{f}=\Omega^{f}(0), \hat{\Omega}^{s}=\Omega^{s}(0)$. The fluidstructure interface is $\Gamma(t)=\partial \Omega^{f}(t) \cap \partial \Omega^{s}(t)$, i.e. the common boundary between $\Omega^{s}(t)$ and $\Omega^{f}(t)$.

A fluid-structure problem is defined by a set of governing equations to be fulfilled in the fluid domain and in the domain of the structure, plus suitable transmission conditions ensuring the continuity of velocity and normal stress across the fluid-structure interface. From the numerical viewpoint, fluid-structure interactions require the solution of coupled fluid and structure models $[17,22,18,19]$ and the stability of the numerical simulations relies on the accuracy of the coupled problem solved at each time-step. A key role is played by the transmission relations which couple at each time level the two physically independent subproblems (fluid and structure). A solution algorithm which enforces the discrete counterpart of both transmission conditions (kinematic and dynamic) is said to be strongly coupled. Hence, strongly coupled methods are generally stable in the energy norm. When the coupling conditions are not exactly satisfied at each time-step, a scheme is called weakly or loosely coupled.

A great variety of strategies have been proposed to solve fluid-structure problems. A first classification relies on the kind of software adopted. When the fluid and the structure sub-problems are solved in a unique solver, the method is said to be monolithic or direct. A monolithic method is strongly coupled by construction, however this approach requires the development of a specific software and lacks of modularity. The examples of monolithic methods are far too numerous to be cited all. We just refer to some recent works $[31,16]$ and references therein.

When the fluid and the structure are solved with two different codes, the result is a partitioned procedure. A partitioned scheme can be either weakly or strongly coupled. In order for a partitioned scheme to be strongly coupled, subiterations need to be performed at each time-step to enforce the transmission conditions with high accuracy. Nevertheless, partitioned procedures are often used to implement weakly coupled schemes. This strategy allows to use optimized methods for each sub-problem. Examples of partitioned procedures can be found in $[25,24]$. 
In this paper, our primary application concerns the simulation of the mechanical interaction between the blood and the wall of large arteries. Dealing with the internal flow of an incompressible fluid whose density is close to the structure density, this class of problems differs from those encountered in other fields (like aeroelasticity) from the numerical point of view. In these cases classical weakly coupled schemes are apparently unstable (see, e.g., [17, 22, 2]), irrespectively of the choice of the time-step. The incompressibility of the fluid and the added-mass effect of the fluid on the structure [17, 2] seem to play an important role in the observed instabilities.

Up to now, strongly coupled schemes have been the only way of avoiding those numerical instabilities. For a partitioned approach, enforcing the transmission conditions requires many sub-iterations and this may lead to prohibitive computational costs. For this reason, over the last years many efforts have been devoted to set-up efficient methods to solve the non-linear implicit coupled problem.

Standard and simple strategies are fixed-point based methods [3]. Unfortunately, even if many acceleration strategies may improve their efficiency $([20,21]$, for example), these methods are very expensive and in some cases may fail to converge $[22,2]$.

Further advances suggest the use of Newton based methods for their fast convergence. They rely on the evaluation of the Jacobian associated to the fluid-solid coupled state. Inexact Newton methods (see, e.g., [11, 35, 12, 36]) and exact Newton algorithms, including differentiation with respect to the fluid domain $[8,9]$, have been proposed.

Another strategy that stems from domain decomposition techniques exploits the physically decoupled structure of the problem itself and allows to obtain the solution through a sequence of independent solves involving separately each sub-problem $[17,6,5]$.

Despite the significant achievements of all these strategies, to our knowledge none of them is able to circumvent strong coupling without compromising stability. In this respect, a relevant improvement is the introduction of a semiimplicit scheme in [7]: although not strongly coupled, it exhibits very good stability properties. This is due to the fact that the pressure stress is coupled implicitly, while the remaining terms of the fluid equations (dissipation, convection and geometrical non-linearities) are explicitly coupled to the structure. In fact, [2] suggests that the explicit treatment of the added-mass term yields instabilities. Therefore the idea of coupling implicitly only the pressure reduces drastically the computational costs without affecting too much the stability.

The algorithm we propose in this work is based on the same idea but we perform the implicit-explicit splitting using an algebraic fractional-step method instead of a differential one. In [7] the coupled system of equations is solved through the Chorin-Temam projection scheme [4, 34]. The accuracy of projection methods depends strongly on the boundary conditions chosen for the differential sub-problems in which the original problem is split. We adopt an algebraic 
fractional scheme called Yosida method, proposed for a pure fluid problem in $[27,28]$. Algebraic fractional-step methods are based on an algebraic decomposition of the matrix arising from the full discretization (both in time and space) of all the equations. In this approach the boundary conditions are incorporated in the discretized operator and no further boundary conditions have to be selected. For a pure fluid problem, the Yosida method differs from the algebraic version of the Chorin-Temam method basically for fulfilling the discrete momentum equation for the fluid, while the Chorin-Temam method guarantees the conservation of the mass.

The resulting algorithm shares the same advantages and drawbacks of the scheme proposed in [7]. In fact, adopting a fractional scheme introduces a splitting error in the solution but greatly simplifies the implementation, especially in comparison to Newton based methods. Moreover our method, being only semi-implicit, does not conserve energy from a theoretical viewpoint but has the great advantage of enhancing efficiency, if compared to any strongly coupled algorithms. In spite of the imperfect energy balance, the scheme is numerically stable for a reasonable range of discretization parameters, especially for those for which explicit methods are known to be unstable.

The scheme we propose is just a specific example of a more general approach exploiting algebraic fractional-step methods to solve fluid-structure interaction problems. The basic idea is to take advantage of the good accuracy and stability properties shown by many algebraic fractional-step schemes (which do not have a differential counterpart $[13,27,28,14]$ ) when solving the incompressible time-dependent Navier-Stokes equations. To our knowledge it is the first time that algebraic fractional-step methods are applied to a coupled fluid-structure problem.

An outline of this report is as follows. In Section 2 we introduce a general fluid-structure interaction problem, describe its associated mathematical model and discretize the resulting system in time and space. We also briefly recall the Yosida fractional-step method. Section 3 presents the new coupling scheme based on the Yosida method. The numerical results obtained by applying this new scheme to a simplified problem are presented in Section 4. Finally, some conclusions and further developments are drawn in Section 5.

\section{Problem setting}

When considering a Lagrangian approach for the structure, the motion of the solid medium is described in terms of its displacement $\boldsymbol{\eta}$ through a smooth injective mapping:

$$
\mathcal{L}^{s}: \hat{\Omega}^{s} \times[0, T] \rightarrow \Omega^{s}(t)
$$

which represents the deformation. Let $\mathbf{F}^{s}(\hat{\boldsymbol{x}}, t)=\nabla_{\hat{\boldsymbol{x}}} \mathcal{L}^{s}(\hat{\boldsymbol{x}}, t)$ be the corresponding deformation gradient and $J^{s}(\hat{\boldsymbol{x}}, t)$ its determinant. We introduce the second 


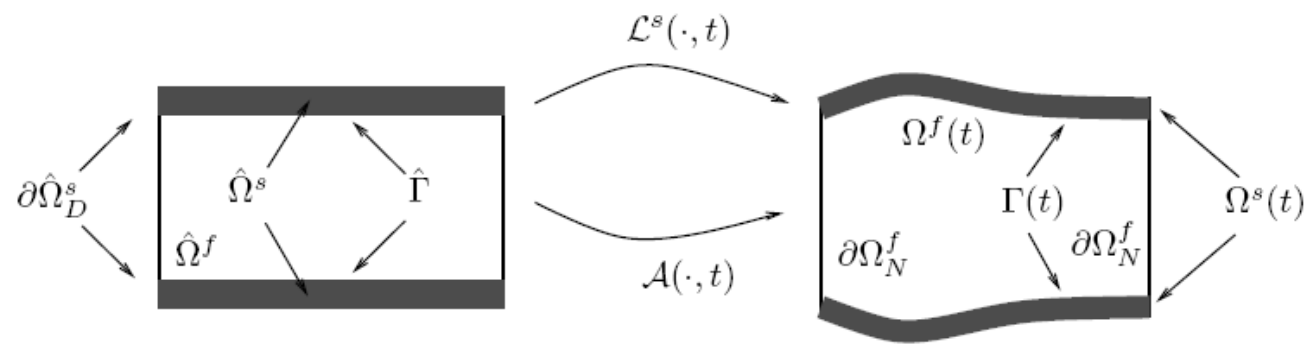

Figure 1: Parametrization of the fluid and structure domains.

Piola-Kirchhoff tensor:

$$
\boldsymbol{\Sigma}=J^{s}\left(\mathbf{F}^{s}\right)^{-1} \boldsymbol{\sigma}^{s}\left(\mathbf{F}^{s}\right)^{-T} .
$$

The solid is assumed to be a hyper-elastic material, characterized by a precise constitutive law relating $\boldsymbol{\Sigma}$ to $\boldsymbol{\eta}$.

We adopt an ALE (Arbitrary Lagrangian Eulerian) description of motion for the fluid, i. e. we parametrize the motion of the actual computational fluid volume $\Omega^{f}(t)$ by an ALE map:

$$
\mathcal{A}: \hat{\Omega}^{f} \times[0, T] \rightarrow \Omega^{f}(t) .
$$

The corresponding deformation gradient is $\mathbf{F}^{f}(\hat{\boldsymbol{x}}, t)=\nabla_{\hat{\boldsymbol{x}}} \mathcal{A}(\hat{\boldsymbol{x}}, t)$ and the determinant is $J^{f}(\hat{\boldsymbol{x}}, t)$. We indicate with $\mathbf{u}(\boldsymbol{x}, t)$ the fluid velocity at a point $\boldsymbol{x}$ and we define the velocity of the computational fluid volume, or ALE velocity, by:

$$
\hat{\mathbf{w}}(\hat{\boldsymbol{x}}, t)=\frac{\partial \mathcal{A}}{\partial t}(\hat{\boldsymbol{x}}, t) .
$$

We assume the fluid to be homogeneous, Newtonian and incompressible, thus the stress tensor reads:

$$
\boldsymbol{\sigma}^{f}(\mathbf{u}, p)=-p \mathbf{I}+2 \mu \boldsymbol{\epsilon}(\mathbf{u}),
$$

where $p$ is the pressure and

$$
\boldsymbol{\epsilon}(\mathbf{u})=\frac{1}{2}\left(\nabla \mathbf{u}+(\nabla \mathbf{u})^{T}\right)
$$

is the strain rate tensor. It is useful to describe the ALE map in terms of the fluid domain displacement $\hat{\mathbf{d}}(\hat{\boldsymbol{x}}, t)=\mathcal{A}(\hat{\boldsymbol{x}}, t)-\hat{\boldsymbol{x}}$. Since the fluid is viscous, $\boldsymbol{\eta}_{\mid \hat{\Gamma}}$ provides the value of $\hat{\mathbf{d}}$ on the interface (condition of fluid adherence). Inside $\hat{\Omega}^{f}$ the displacement $\hat{\mathbf{d}}(\hat{\boldsymbol{x}}, t)$ is arbitrary: it can be any reasonable extension of $\boldsymbol{\eta}_{\mid \hat{\Gamma}}$ over $\hat{\Omega}^{f}$. We will denote the extension operator by Ext. Through $\hat{\mathbf{d}}$ we calculate the current fluid domain $\Omega^{f}(t)=(I+\hat{\mathbf{d}})\left(\hat{\Omega}^{f}\right)$. 
The fluid-structure problem that we will consider couples the incompressible Navier-Stokes equations in conservative ALE form to the elastodynamics equation for a hyper-elastic solid. Thus it reads:

find $\hat{\mathbf{d}}: \hat{\Omega}^{f} \times[0, T] \rightarrow \mathbb{R}^{d}, \mathbf{u}: \mathcal{T}_{\mathcal{A}} \rightarrow \mathbb{R}^{d}, p: \mathcal{T}_{\mathcal{A}} \rightarrow \mathbb{R}$ and $\boldsymbol{\eta}: \hat{\Omega}^{s} \times[0, T] \rightarrow \mathbb{R}^{d}$ such that

- equations for the geometry:

$$
\hat{\mathbf{d}}=\operatorname{Ext}\left(\boldsymbol{\eta}_{\mid \hat{\Gamma}}\right), \quad \hat{\mathbf{w}}=\frac{\partial \hat{\mathbf{d}}}{\partial t}, \quad \Omega^{f}(t)=(I+\hat{\mathbf{d}})\left(\hat{\Omega}^{f}\right),
$$

- equations for the fluid:

$$
\begin{array}{ll}
\frac{1}{J^{f}}{\left.\frac{\partial J^{f} \mathbf{u}}{\partial t}\right|_{\hat{\boldsymbol{x}}}}+\operatorname{div}\left(\mathbf{u} \otimes(\mathbf{u}-\mathbf{w})-\frac{1}{\rho^{f}} \boldsymbol{\sigma}^{f}(\mathbf{u}, p)\right)=\mathbf{0}, & \text { in } \Omega^{f}(t), \\
\operatorname{div} \mathbf{u}=0, & \text { in } \Omega^{f}(t), \\
\mathbf{u}=\mathbf{u}_{D}, & \text { on } \partial \Omega_{D}^{f}, \\
\boldsymbol{\sigma}^{f}(\mathbf{u}, p) \cdot \mathbf{n}^{f}=\mathbf{g}_{N}^{f}, & \text { on } \partial \Omega_{N}^{f}, \\
\mathbf{u}=\mathbf{w}, & \text { on } \Gamma(t),
\end{array}
$$

- equations for the solid structure:

$$
\begin{array}{ll}
\rho^{s} \frac{\partial^{2} \boldsymbol{\eta}}{\partial t^{2}}-\operatorname{div}_{\hat{\boldsymbol{x}}}\left(\mathbf{F}^{s} \boldsymbol{\Sigma}\right)=\mathbf{0}, & \text { in } \hat{\Omega}^{s}, \\
\boldsymbol{\eta}=\mathbf{0}, & \text { on } \partial \hat{\Omega}_{D}^{s}, \\
\mathbf{F}^{s} \boldsymbol{\Sigma} \cdot \mathbf{n}^{s}=J^{s}\left|\left(\mathbf{F}^{s}\right)^{-T} \mathbf{n}^{s}\right| \mathbf{g}_{N}^{s}, & \text { on } \partial \hat{\Omega}_{N}^{s}, \\
\mathbf{F}^{s} \boldsymbol{\Sigma} \cdot \mathbf{n}^{s}=J^{s} \hat{\boldsymbol{\sigma}}^{f}(\mathbf{u}, p)\left(\mathbf{F}^{s}\right)^{-T} \mathbf{n}^{s}, & \text { on } \hat{\Gamma} .
\end{array}
$$

The first equality in (2) is the geometry coupling condition, i.e. continuity of displacement at $\Gamma$. Boundary condition $(3)_{5}$ imposes the continuity of velocities at the interface. In order to ensure the balance of stresses on the interface, we enforce the continuity of stresses through $(4)_{4}$. These are the three interface coupling conditions.

\subsection{Variational formulation}

In order to reformulate the coupled problem in a weak form, we need a series of notations. We indicate with $H^{m}(\Omega), m>0$, the usual Sobolev spaces for a given domain $\Omega \subset \mathbb{R}^{d}$. The scalar product in $L^{2}(\Omega)=H^{0}(\Omega)$ is denoted by $(\cdot, \cdot)_{\Omega}$. 
To simplify the setting up of the variational formulation of the problem, we choose $\partial \Omega^{f}(t) \backslash \Gamma(t)=\partial \Omega_{N}^{f}(t)$, see Fig. 1. We define the following spaces of test functions:

$$
V^{f}=\left\{\mathbf{v}^{f} \in\left[H^{1}\left(\Omega^{f}\right)\right]^{d}: \mathbf{v}^{f}=\mathbf{0} \text { on } \Gamma\right\},
$$

and

$$
\hat{V}^{s}=\left\{\hat{\mathbf{v}}^{s} \in\left[H^{1}\left(\hat{\Omega}^{s}\right)\right]^{d-1}: \hat{\mathbf{v}}^{s}=\mathbf{0} \text { on } \partial \hat{\Omega}_{D}^{s}\right\} .
$$

From now on we will consider a $(d-1)$-dimensional structure and so $\overline{\Omega^{s}}=\Gamma$. All the results we will find in what follows can be generalized, with proper adaptations, to the case of $d$-dimensional structure.

We write the velocity as $\mathbf{u}=\mathbf{u}_{0}+\mathbf{u}_{\Gamma}$, with $\mathbf{u}_{0}=\mathbf{0}$ on $\Gamma$ and $\mathbf{u}_{\Gamma}=\frac{\partial \boldsymbol{\eta}}{\partial t}$ on $\Gamma$, that is $\mathbf{u}_{\Gamma}$ satisfies the coupling condition $(3)_{5}$.

By multiplying the fluid equations $(3)_{1,2}$ by $\mathbf{v}^{f}$, after integration by parts and taking into account the boundary conditions, we get the following variational formulation of the fluid sub-problem:

Find $\mathbf{u}_{0} \in V^{f}, p \in L^{2}\left(\Omega^{f}\right)$ for all $t \in \mathbb{R}^{+}$, such that:

$$
\begin{array}{ll}
\frac{d}{d t}\left(\left(\mathbf{u}_{0}+\mathbf{u}_{\Gamma}\right), \mathbf{v}^{f}\right)_{\Omega^{f}(t)}+\left(\operatorname{div}\left(\left(\mathbf{u}_{0}+\mathbf{u}_{\Gamma}\right) \otimes\left(\mathbf{u}_{0}+\mathbf{u}_{\Gamma}-\mathbf{w}\right)\right), \mathbf{v}^{f}\right)_{\Omega^{f}(t)}+ & \\
\frac{1}{\rho^{f}}\left(\left(\boldsymbol{\sigma}^{f}\left(\mathbf{u}_{0}+\mathbf{u}_{\Gamma}, p\right), \nabla \mathbf{v}^{f}\right)\right)_{\Omega^{f}(t)}=\frac{1}{\rho^{f}}\left(\mathbf{g}_{N}^{f}, \mathbf{v}^{f}\right)_{\Omega_{N}^{f}(t)}, & \forall \mathbf{v}^{f} \in V^{f}, \quad \\
\left(q, \operatorname{div} \mathbf{u}_{0}\right)_{\Omega^{f}(t)}+\left(q, \operatorname{div} \mathbf{u}_{\Gamma}\right)_{\Omega^{f}(t)}=0, & \forall q \in L^{2}\left(\Omega^{f}(t)\right) .
\end{array}
$$

On the other hand, by multiplying $(4)_{1}$ by $\hat{\mathbf{v}}^{s}$ and after integration by parts, it follows that the variational formulation for solid sub-problem is:

Find $\boldsymbol{\eta} \in \hat{V}^{s}$ such that

$$
\begin{aligned}
& \left(\rho^{s} \frac{\partial^{2} \boldsymbol{\eta}}{\partial t^{2}}, \hat{\mathbf{v}}^{s}\right)_{\hat{\Omega}^{s}}+\left(\mathbf{F}^{s} \boldsymbol{\Sigma}, \boldsymbol{d i v}_{\hat{\boldsymbol{x}}} \hat{\mathbf{v}}^{s}\right)_{\hat{\Omega}^{s}}= \\
& \quad\left(J^{s}\left|\left(\mathbf{F}^{s}\right)^{-T} \mathbf{n}^{s}\right| \mathbf{g}_{N}^{s}, \hat{\mathbf{v}}^{s}\right)_{\partial \hat{\Omega}_{N}^{s}}-\left(\boldsymbol{\sigma}^{f}(\mathbf{u}, p) \mathbf{n}^{f}, \mathbf{v}^{s}\right)_{\Gamma(t)}, \quad \forall \hat{\mathbf{v}}^{s} \in \hat{V}^{s} .
\end{aligned}
$$

The fluid interface load can be seen as the variational residual of (5) when the test functions are not vanishing at the interface; indeed,

$$
\begin{gathered}
\left(\boldsymbol{\sigma}^{f}(\mathbf{u}, p) \mathbf{n}^{f}, \mathbf{v}^{s}\right)_{\Gamma(t)}=\rho^{f} \frac{d}{d t}\left(\mathbf{u}, \mathcal{E} \mathbf{v}^{s}\right)_{\Omega^{f}(t)}+\rho^{f}\left(\operatorname{div}(\mathbf{u} \otimes(\mathbf{u}-\mathbf{w})), \mathcal{E} \mathbf{v}^{s}\right)_{\Omega^{f}(t)}+ \\
\left(\left(\boldsymbol{\sigma}^{f}(\mathbf{u}, p), \nabla \mathcal{E} \mathbf{v}^{s}\right)\right)_{\Omega^{f}(t)}, \quad \forall \mathbf{v}^{s} \in V^{s},
\end{gathered}
$$

where the operator $\mathcal{E}$ represents a continuous extension operator from $V^{s}$ into the space $W^{f}=\left\{\mathbf{v}^{f} \in\left[H^{1}\left(\Omega^{f}\right)\right]^{d}: \mathbf{v}^{f}=\mathbf{0}\right.$ on $\left.\partial \Omega^{f}(t) \backslash \Gamma(t)\right\}$. 


\subsection{Space and time discretization: the fully discrete problem}

Let us introduce a triangulation $\hat{\mathcal{T}}_{h}[30]$ of the reference configuration $\hat{\Omega}$ and its image $\mathcal{T}_{h}$ through a discrete ALE mapping $\mathcal{A}_{h, t}$. As customary, $h$ represents the maximum size of the elements of $\hat{\mathcal{T}}_{h}$. The triangulation $\mathcal{T}_{h}$ induces a partition $\mathcal{I}_{h}$ on $\Gamma(t)$. Let $\mathcal{I}_{H}$ be an independent partition of $\Gamma(t)$ whose maximum size of the elements is $H$. Being the diameters of the general elements of the two meshes, $h, H$ refer to the level of refinement of the triangulations. We also assume the triangulations to be quasi-uniform [30]. For the sake of simplicity, we will consider the case of geometric conforming meshes, i.e. $\mathcal{I}_{h} \equiv \mathcal{I}_{H}$.

Let $V_{h}^{f} \subset\left[H^{1}\left(\Omega^{f}\right)\right]^{d}, Q_{h}^{f} \subset L^{2}\left(\Omega^{f}\right)$ and $\hat{V}_{H}^{s} \subset \hat{V}^{s}$ be the finite element spaces approximating the corresponding continuous ones [30]. We introduce the Lagrange basis $\left\{\boldsymbol{\phi}_{i}\right\}_{i=1}^{\mathcal{N}_{v}} \oplus\left\{\boldsymbol{\phi}_{j}^{b}\right\}_{j=1}^{\mathcal{N}_{v}^{b}},\left\{\pi_{i}\right\}_{i=1}^{\mathcal{N}_{p}}$ and $\left\{\boldsymbol{\psi}_{i}\right\}_{i=1}^{\mathcal{N}_{s}}$ associated, respectively, to $V_{h}^{f}, Q_{h}^{f}$ and $\hat{V}_{H}^{s}$. The set $\left\{\boldsymbol{\phi}_{j}^{b}\right\}_{j=1}^{\mathcal{N}_{v}^{b}}$ corresponds to the nodes on $\Gamma(t)$. The fluid sub-problem requires that the pair $\left(Q_{h}^{f}, V_{h}^{f}\right)$ satisfy the inf-sup condition:

$$
\inf _{q_{h} \in Q_{h}^{f} \mathbf{v}_{h}^{f} \in V_{h}^{f}} \frac{\int_{\Omega^{f}} \operatorname{div} \mathbf{v}_{h}^{f} q_{h} d \Omega}{\left\|\mathbf{v}_{h}^{f}\right\|_{H^{1}\left(\Omega^{f}\right)}\left\|q_{h}\right\|_{L^{2}\left(\Omega^{f}\right)}} \geq \beta,
$$

where the constant $\beta>0$ is independent of $h([1,30])$. We remind that this property is necessary for the well posedness of the discrete problem.

Moreover, let $\mathcal{E}_{h}$ be a discrete extension operator from $\hat{V}_{H}^{s}$ into $V_{h}^{f}$.

We can write the finite element approximation of the unknowns:

$$
\mathbf{u}_{h}(\boldsymbol{x}, t)=\sum_{i=0}^{N_{v}} U_{0_{i}}(t) \boldsymbol{\phi}_{i}(\boldsymbol{x}, t)+\sum_{j=0}^{N_{v}^{b}} U_{\Gamma_{j}}(t) \boldsymbol{\phi}_{j}^{b}(\boldsymbol{x}, t), p_{h}(\boldsymbol{x}, t)=\sum_{k=0}^{N_{p}} P_{k}(t) \pi_{k}(\boldsymbol{x}, t),
$$

where the nodal values $U_{\Gamma_{j}}$ depend on the structure velocity. We also set:

$$
\boldsymbol{\eta}_{H}(\hat{\boldsymbol{x}}, t)=\sum_{i=0}^{N_{s}} \eta_{i}(t) \boldsymbol{\psi}_{i}(\hat{\boldsymbol{x}}), \dot{\boldsymbol{\eta}}_{H}(\hat{\boldsymbol{x}}, t)=\sum_{j=0}^{N_{s}} \dot{\eta}_{j}(t) \boldsymbol{\psi}_{j}(\hat{\boldsymbol{x}})
$$

Let $\mathbf{U}_{0}, \mathbf{P}, \mathbf{U}_{\Gamma}, \boldsymbol{\eta}$ and $\dot{\boldsymbol{\eta}}$ be the vectors of the coefficients of the linear combinations (8) and (9).

We divide the time interval $[0, T]$ using a time-step $\delta t$ and solve the problem at each time level $t^{n}=n \cdot \delta t$, with $n=1, \ldots, N$ and $N=T / \delta t$.

There are many strategies for discretising in time the coupled fluid-structure problem. Here, for the sake of simplicity, we employ the implicit Euler scheme for the fluid and the mid-point rule for the structure. The latter applied to the 
structure equation after being discretized in space reads:

$$
\begin{array}{lr}
\rho^{s}\left(\frac{\dot{\boldsymbol{\eta}}_{H}^{n+1}-\dot{\boldsymbol{\eta}}_{H}^{n}}{\delta t}, \hat{\mathbf{v}}_{H}^{s}\right)_{\hat{\Omega}^{s}}+\frac{1}{2}\left(\mathbf{F}^{s, n+1} \boldsymbol{\Sigma}\left(\boldsymbol{\eta}_{H}^{n+1}\right)+\mathbf{F}^{s, n} \boldsymbol{\Sigma}\left(\boldsymbol{\eta}_{H}^{n}\right), \boldsymbol{d i v}_{\hat{\boldsymbol{x}}} \hat{\mathbf{v}}_{H}^{s}\right)_{\hat{\Omega}^{s}}= \\
-\left(\boldsymbol{\sigma}^{f}\left(\mathbf{u}_{h}^{n+1}, p_{h}^{n+1}\right) \mathbf{n}^{f}, \mathbf{v}_{H}^{s}\right)_{\Gamma(t)}, & \forall \hat{\mathbf{v}}_{H}^{s} \in \hat{V}_{H}^{s}, \\
\left(\frac{\dot{\boldsymbol{\eta}}_{H}^{n+1}+\dot{\boldsymbol{\eta}}_{H}^{n}}{2}, \hat{\mathbf{v}}_{H}^{s}\right)_{\hat{\Omega}^{s}}=\left(\frac{\boldsymbol{\eta}_{H}^{n+1}-\boldsymbol{\eta}_{H}^{n}}{\delta t}, \hat{\mathbf{v}}_{H}^{s}\right)_{\hat{\Omega}^{s}}, & \forall \hat{\mathbf{v}}_{H}^{s} \in \hat{V}_{H}^{s} .
\end{array}
$$

The superscript $n$ denotes the approximation of the unknown at time $t^{n}$. We replace $\mathbf{u}_{\Gamma_{h}}^{n+1}$ with its first order discretization, so that $\mathbf{U}_{\Gamma}^{n+1}=\left(\boldsymbol{\eta}^{n+1}-\boldsymbol{\eta}^{n}\right) / \delta t$.

In the sequel, we will consider the linearized fluid structure problem: we linearize the non-linear convective term in $(3)_{1}$ around $\mathbf{u}^{*}=\mathbf{u}_{h}^{n}$ and $\boldsymbol{\sigma}^{s}$ in (1) stands for the linearized stress tensor. To simplify the problem without compromising its generality, we assume $\mathbf{g}_{N}^{s}=\mathbf{0}$.

In order to write the fully discretized coupled problem, we set:

- the mass matrices: $M_{i, j}^{f}=\int_{\Omega^{f, n+1}} \phi_{j} \phi_{i}, M_{i, j}^{s f}=\int_{\Omega^{f, n+1}} \phi_{j} \mathcal{E}_{h} \psi_{i}, M_{i, j}^{s b}=$ $\int_{\Omega^{f, n+1}} \phi_{j}^{b} \mathcal{E}_{h} \boldsymbol{\psi}_{i}, M_{i, j}^{s}=\int_{\hat{\Omega}^{s}} \boldsymbol{\psi}_{j} \boldsymbol{\psi}_{i}$

- the matrices $C$ :

$$
\begin{aligned}
& C_{i, j}^{f}=\frac{1}{\delta t} M_{i, j}^{f}+\nu \int_{\Omega^{f, n+1}} \nabla \boldsymbol{\phi}_{j}: \nabla \boldsymbol{\phi}_{i}+\int_{\Omega^{f, n+1}}\left[\left(\mathbf{u}^{*}-\mathbf{w}_{h}^{n+1}\right) \cdot \nabla\right] \boldsymbol{\phi}_{j} \cdot \boldsymbol{\phi}_{i} \\
& C_{i, j}^{s f}=\frac{1}{\delta t} M_{i, j}^{s f}+\nu \int_{\Omega^{f, n+1}} \nabla \boldsymbol{\phi}_{j}: \nabla \mathcal{E}_{h} \boldsymbol{\psi}_{i}+\int_{\Omega}^{f, n+1}\left[\left(\mathbf{u}^{*}-\mathbf{w}_{h}^{n+1}\right) \cdot \nabla\right] \boldsymbol{\phi}_{j} \cdot \mathcal{E}_{h} \boldsymbol{\psi}_{i}, \\
& C_{i, j}^{s b}=\frac{1}{\delta t} M_{i, j}^{s b}+\nu \int_{\Omega^{f, n+1}} \nabla \boldsymbol{\phi}_{j}^{b}: \nabla \mathcal{E}_{h} \boldsymbol{\psi}_{i}+\int_{\Omega^{f, n+1}}\left[\left(\mathbf{u}^{*}-\mathbf{w}_{h}^{n+1}\right) \cdot \nabla\right] \boldsymbol{\phi}_{j}^{b} \cdot \mathcal{E}_{h} \boldsymbol{\psi}_{i}
\end{aligned}
$$

- the matrices $B: B_{i, j}^{f}=-\int_{\Omega^{f, n+1}}\left(\operatorname{div} \phi_{j}\right) \pi_{i}, B_{i, j}^{s}=-\int_{\Omega^{f, n+1}}\left(\operatorname{div} \mathcal{E}_{h} \psi_{j}\right) \pi_{i}$;

- the matrix $K^{s}$ and $D: K_{i, j}^{s}=\frac{\delta t}{2 \rho^{f}}\left(\int_{\hat{\Omega}^{s}} \mathbf{F}^{s, n+1} \boldsymbol{\Sigma}^{n+1}\left(\boldsymbol{\psi}_{j}\right): \operatorname{div} \boldsymbol{\psi}_{i}\right)$,

$$
D=\frac{2}{\delta t} \frac{\rho^{s}}{\rho^{f}} M^{s}+K^{s}+C^{s b}
$$

- the vectors $\mathbf{F}_{N}: \mathbf{F}_{N_{i}}^{f}=\frac{1}{\rho^{f}} \int_{\Omega^{f, n+1}} \mathbf{g}_{N}^{f} \boldsymbol{\phi}_{i}, \mathbf{F}_{N_{j}}^{s}=\frac{1}{\rho^{f}} \int_{\Omega^{f, n+1}} \mathbf{g}_{N}^{f} \boldsymbol{\phi}_{j}^{b}$.

Then we use the shortened notations for the matrix transposes: $M^{f s}=\left(M^{s f}\right)^{T}$ and $C^{f s}=\left(C^{s f}\right)^{T}$.

Except for $M^{s}$, all the matrices need to be calculated at each time $t^{n+1}$, yet we drop the superscript $n+1$ for ease of notation. For the discretization of the time derivatives we also need some matrices at time $t^{n}$ : those will be named with the superscript $n$.

It is worthwhile pointing out that the fluid and fluid-structure matrices are calculated over $\Omega^{f, n+1}=\Omega^{f}\left(t^{n+1}\right)$, which depends on the unknown $\boldsymbol{\eta}_{H}^{n+1}$ through the ALE velocity $\mathbf{w}_{h}^{n+1}(2)$.

Then at each time level $t^{n+1}$, the discretization in time yields the system:

$$
A^{n+1} \mathbf{X}^{n+1}=\mathbf{F}^{n+1}
$$


where

$$
A^{n+1}=\left[\begin{array}{ccc}
C^{f} & \left(B^{f}\right)^{T} & C^{f s} \\
B^{f} & 0 & B^{s} \\
C^{s f} & \left(B^{s}\right)^{T} & D
\end{array}\right], \mathbf{X}^{n+1}=\left[\begin{array}{c}
\mathbf{U}_{0}^{n+1} \\
\mathbf{P}^{n+1} \\
\mathbf{U}_{\Gamma}^{n+1}
\end{array}\right]
$$

and

$$
\mathbf{F}^{n+1}=\left[\begin{array}{c}
\mathbf{F}_{1}^{n+1} \\
\mathbf{F}_{2}^{n+1} \\
\mathbf{F}_{3}^{n+1}
\end{array}\right], \begin{aligned}
& \mathbf{F}_{1}^{n+1}=\mathbf{F}_{N}^{f}+\frac{1}{\delta t} M^{f, n} \mathbf{U}^{n} \\
& \quad \mathbf{F}_{3}^{n+1}=\mathbf{0}=\mathbf{F}_{N}^{s}+\frac{2}{\delta t} K^{s, n} \boldsymbol{\eta}^{n}+\frac{2}{\delta t} \frac{\rho^{s}}{\rho^{f}} M^{s} \dot{\boldsymbol{\eta}}^{n} .
\end{aligned}
$$

Once $\mathbf{U}_{\Gamma}^{n+1}$ is known, the structure displacement and velocity nodal values can be retrieved by explicit calculations:

$$
\boldsymbol{\eta}^{n+1}=\delta t \mathbf{U}_{\Gamma}^{n+1}+\boldsymbol{\eta}^{n}, \dot{\boldsymbol{\eta}}^{n+1}=2 \mathbf{U}_{\Gamma}^{n+1}-\dot{\boldsymbol{\eta}}^{n} .
$$

To summarize, we describe the procedure above as follows: given the initial solution $\mathbf{u}_{h 0}, p_{h 0}, \boldsymbol{\eta}_{H 0}$ and $\dot{\boldsymbol{\eta}}_{H 0}$, for every $n \geq 0$, find $\mathbf{u}_{h}^{n+1} \in V_{h}^{f}, p_{h}^{n+1} \in Q_{h}^{f}$, $\boldsymbol{\eta}_{H}^{n+1}, \dot{\boldsymbol{\eta}}_{H}^{n+1} \in \hat{V}_{H}^{s}$ by solving system (11) and the equations in (12) to get the nodal values to be used in the linear combinations (8) and (9).

\subsection{The exact $L U$ factorization of the coupled system}

To solve system (11) one could use a global approach such as a preconditioned Krylov method (with proper choice of preconditioner).

Alternatively, system (11) can be solved by a block $L U$ factorization of $A^{n+1}$, that is

$$
A^{n+1}=L U .
$$

In order to find the two factors $L$ and $U$, we introduce the matrix:

$$
S:=-B^{f}\left(C^{f}\right)^{-1}\left(B^{f}\right)^{T}
$$

called pressure Schur complement since it is the Schur complement of $C^{f}$ in the following submatrix of $A^{n+1}$ :

$$
A_{0}=\left[\begin{array}{cc}
C^{f} & \left(B^{f}\right)^{T} \\
B^{f} & 0
\end{array}\right]
$$

that corresponds to the pure fluid discretization. In analogy, we write the Schur complements

$$
\begin{aligned}
& S_{1}:=B^{s}-B^{f}\left(C^{f}\right)^{-1} C^{f s}, \\
& S_{2}:=\left(B^{s}\right)^{T}-C^{s f}\left(C^{f}\right)^{-1}\left(B^{f}\right)^{T}, \\
& S_{3}:=D-C^{s f}\left(C^{f}\right)^{-1} C^{f s},
\end{aligned}
$$

respectively related to the following submatrices of $A^{n+1}$ :

$$
A_{1}=\left[\begin{array}{cc}
C^{f} & C^{f s} \\
B^{f} & B^{s}
\end{array}\right], A_{2}=\left[\begin{array}{cc}
C^{f} & \left(B^{f}\right)^{T} \\
C^{s f} & \left(B^{s}\right)^{T}
\end{array}\right], A_{3}=\left[\begin{array}{cc}
C^{f} & C^{f s} \\
C^{s f} & D
\end{array}\right]
$$


The factors $L$ and $U$ in (13) have the following form:

$$
L=\left[\begin{array}{ccc}
C^{f} & 0 & 0 \\
B^{f} & S & 0 \\
C^{s f} & S_{2} & S_{3}-S_{2} S^{-1} S_{1}
\end{array}\right], U=\left[\begin{array}{ccc}
I & \left(C^{f}\right)^{-1}\left(B^{f}\right)^{T} & \left(C^{f}\right)^{-1} C^{f s} \\
0 & I & S^{-1} S_{1} \\
0 & 0 & I
\end{array}\right]
$$

Solving system (11) through the block $L U$ factorization (13) involves the solution of the following subsystems:

$$
\begin{aligned}
& L \text { - step: find } \widetilde{\mathbf{U}}_{0}^{n+1}, \widetilde{\mathbf{P}}^{n+1}, \widetilde{\mathbf{U}}_{\Gamma}^{n+1} \text { : } \\
& C^{f} \widetilde{\mathbf{U}}_{0}^{n+1}=\mathbf{F}_{1}^{n+1} \\
& B^{f} \widetilde{\mathbf{U}}_{0}^{n+1}+S \widetilde{\mathbf{P}}^{n+1}=\mathbf{F}_{2}^{n+1} \\
& C^{s f} \widetilde{\mathbf{U}}_{0}^{n+1}+S_{2} \widetilde{\mathbf{P}}^{n+1}+\left(S_{3}-S_{2} S^{-1} S_{1}\right) \widetilde{\mathbf{U}}_{\Gamma}^{n+1}=\mathbf{F}_{3}^{n+1}, \\
& U \text { - step: find } \mathbf{U}_{0}^{n+1}, \mathbf{P}^{n+1}, \mathbf{U}_{\Gamma}^{n+1} \text { : } \\
& \mathbf{U}_{\Gamma}^{n+1}=\widetilde{\mathbf{U}}_{\Gamma}^{n+1} \\
& \mathbf{P}^{n+1}+S^{-1} S_{1} \mathbf{U}_{\Gamma}^{n+1}=\widetilde{\mathbf{P}}^{n+1} \\
& \mathbf{U}_{0}^{n+1}+\left(C^{f}\right)^{-1}\left(B^{f}\right)^{T} \mathbf{P}^{n+1}+\left(C^{f}\right)^{-1} C^{f s} \mathbf{U}_{\Gamma}^{n+1}=\widetilde{\mathbf{U}}_{0}^{n+1} \text {. }
\end{aligned}
$$

The computational complexity of the $L U$ scheme can be reduced provided $\left(C^{f}\right)^{-1}$ in (14), (15), (16), (17) is replaced by a simpler matrix. This is the basic idea underlying algebraic fractional step algorithm in general, and the Yosida algorithm in particular, see e.g. [28].

\subsection{The inexact $L U$ factorization of the coupled system: the Yosida scheme}

The Yosida method is characterized by replacing the factors $L$ and $U$ of $A^{n+1}$

(13) with an inexact $L U$ factorization of the form $\hat{A}^{n+1}=\hat{L} \hat{U}$. The approximate factors $\hat{L}$ and $\hat{U}$ are determined as follows. First of all we approximate the Schur complements $(14),(15),(16),(17)$ by replacing $\left(C^{f}\right)^{-1}$ with the zero-th order truncation of its Neumann expansion:

$$
\begin{aligned}
& \hat{S}:=-B^{f} \delta t\left(M^{f}\right)^{-1}\left(B^{f}\right)^{T}, \quad \hat{S}_{1}:=B^{s}-B^{f} \delta t\left(M^{f}\right)^{-1} C^{f s}, \\
& \hat{S}_{2}:=\left(B^{s}\right)^{T}-C^{s f} \delta t\left(M^{f}\right)^{-1}\left(B^{f}\right)^{T}, \quad \hat{S}_{3}:=D-C^{s f} \delta t\left(M^{f}\right)^{-1} C^{f s} .
\end{aligned}
$$

Thus we can write the inexact factors as:

$$
\hat{L}=\left[\begin{array}{ccc}
C^{f} & 0 & 0 \\
B^{f} & \hat{S} & 0 \\
C^{s f} & \hat{S}_{2} & \hat{S}_{3}-\hat{S}_{2} \hat{S}^{-1} \hat{S}_{1}
\end{array}\right], \hat{U}=\left[\begin{array}{ccc}
I & \left(C^{f}\right)^{-1}\left(B^{f}\right)^{T} & \left(C^{f}\right)^{-1} C^{f s} \\
0 & I & \hat{S}^{-1} \hat{S}_{1} \\
0 & 0 & I
\end{array}\right]
$$

At each time-step, system (11) is replaced by:

$$
\hat{A}^{n+1} \mathbf{X}^{n+1}=\mathbf{F}^{n+1} \text {. }
$$


The Yosida method computes an approximate solution affected by the splitting error. Indeed, $\mathbf{X}^{n+1}$ in (23) is an approximation of the solution of (11) and the right hand side takes into account the approximated solutions from previous steps. Although they should better be indicated with $\widehat{\mathbf{X}}^{n+1}$ and $\widehat{\mathbf{F}}^{n+1}$, nonetheless we prefer to keep the notation unchanged for simplicity.

Solving system (11) through the inexact factorization $\hat{L} \hat{U}$ at each time level consists in solving the following simpler subsystems (instead of (19), (20)):

$$
\begin{aligned}
& \hat{L} \text { - step: find } \widetilde{\mathbf{U}}_{0}^{n+1}, \widetilde{\mathbf{P}}^{n+1}, \widetilde{\mathbf{U}}_{\Gamma}^{n+1} \text { : } \\
& C^{f} \widetilde{\mathbf{U}}_{0}^{n+1}=\mathbf{F}_{1}^{n+1} \\
& B^{f} \widetilde{\mathbf{U}}_{0}^{n+1}+\hat{S} \widetilde{\mathbf{P}}^{n+1}=\mathbf{F}_{2}^{n+1} \\
& C^{s f} \widetilde{\mathbf{U}}_{0}^{n+1}+\hat{S}_{2} \widetilde{\mathbf{P}}^{n+1}+\left(\hat{S}_{3}-\hat{S}_{2} \hat{S}^{-1} \hat{S}_{1}\right) \widetilde{\mathbf{U}}_{\Gamma}^{n+1}=\mathbf{F}_{3}^{n+1}, \\
& \hat{U} \text { - step: find } \mathbf{U}_{0}^{n+1}, \mathbf{P}^{n+1}, \mathbf{U}_{\Gamma}^{n+1} \text { : } \\
& \mathbf{U}_{\Gamma}^{n+1}=\widetilde{\mathbf{U}}_{\Gamma}^{n+1} \\
& \mathbf{P}^{n+1}+\hat{S}^{-1} \hat{S}_{1} \mathbf{U}_{\Gamma}^{n+1}=\widetilde{\mathbf{P}}^{n+1} \\
& C^{f} \mathbf{U}_{0}^{n+1}+\left(B^{f}\right)^{T} \mathbf{P}^{n+1}+C^{f s} \mathbf{U}_{\Gamma}^{n+1}=C^{f} \widetilde{\mathbf{U}}_{0}^{n+1} .
\end{aligned}
$$

The computational convenience with respect to the exact $L U$ factorization consists in the fact that the approximated Schur complements are considerably easier to solve than the exact ones.

An important issue for differential splitting scheme, such as the ChorinTemam projection method, is the prescription of pressure boundary conditions. In solving a differential problem for the pressure, unphysical boundary conditions are prescribed, which in general induce boundary layers in the behavior of the pressure error. In particular, this can negatively affect the numerical solution of a fluid-structure problem, in which it is important to evaluate at each time-step the normal stress exerted by the fluid on the structure. Algebraic subproblems are easined by the fact that no additional boundary condition on the pressure has to be provided, since this information is intrinsically carried out by the (inexact) factorization. This usually reflects in a better behavior of the pressure on the boundary, as shown in [28] for the case of the Yosida scheme applied to the incompressible Navier-Stokes equations in a domain with rigid boundaries.

\section{A Yosida based semi-implicit coupling}

We rearrange the two steps (24) and (25) in a semi-implicit coupling scheme which will resemble, at the algebraic level, the differential fractional step presented in [7]. Suppose that we know $\Omega^{n}, \mathbf{U}_{0}^{n}, \mathbf{P}^{n}, \mathbf{U}_{\Gamma}^{n}$, and therefore $\boldsymbol{\eta}^{n}, \dot{\boldsymbol{\eta}}^{n}$, at time $t^{n}$; then we calculate $\Omega^{n+1}, \mathbf{U}_{0}^{n+1}, \mathbf{P}^{n+1}, \mathbf{U}_{\Gamma}^{n+1}$ with the following procedure: 
- Step 0: definition of the new domain, by approximating the fluid-structure interface with $\boldsymbol{\eta}^{n}$ :

$$
\hat{\mathbf{d}}^{n+1}=\operatorname{Ext}\left(\boldsymbol{\eta}_{\mid \hat{\Gamma}}^{n}\right), \quad \Omega^{f, n+1}=\hat{\Omega}^{f}+\hat{\mathbf{d}}^{n+1}, \quad \mathbf{w}^{n+1}=\frac{\hat{\mathbf{d}}^{n+1}-\hat{\mathbf{d}}^{n}}{\delta t} ;
$$

- Step 1 (explicit flow computation): computation of the intermediate velocity $\widetilde{\mathbf{U}}_{0}^{n+1}$ :

$$
C^{f} \widetilde{\mathbf{U}}_{0}^{n+1}=\mathbf{F}_{1}^{n+1}
$$

- Step 2 (implicit coupling): computation of pressure $\mathbf{P}^{n+1}$ and interface velocity $\mathbf{U}_{\Gamma}^{n+1}$ :

$$
\begin{aligned}
& \hat{S} \mathbf{P}^{n+1}+\hat{S}_{1} \mathbf{U}_{\Gamma}^{n+1}=\mathbf{F}_{2}^{n+1}-B^{f} \widetilde{\mathbf{U}}_{0}^{n+1}, \\
& \hat{S}_{2} \mathbf{P}^{n+1}+\hat{S}_{3} \mathbf{U}_{\Gamma}^{n+1}=\mathbf{F}_{3}^{n+1}-C^{s f} \widetilde{\mathbf{U}}_{0}^{n+1},
\end{aligned}
$$

and then calculation of structure displacement and velocity through (12);

- Step 3 (explicit flow computation): velocity correction:

$$
C^{f} \mathbf{U}_{0}^{n+1}=C^{f} \widetilde{\mathbf{U}}_{0}^{n+1}-\left(B^{f}\right)^{T} \mathbf{P}^{n+1}-C^{f s} \mathbf{U}_{\Gamma}^{n+1} .
$$

The equation $(28)_{1}$ is obtained by combining $(24)_{2}$ and $(25)_{2}$, whereas equation $(28)_{2}$ is given by the combination of $(24)_{3}$ and $(25)_{2}$.

At each time-step we have to solve three linear systems; two of them (Step 1 and 3 ) share the same matrix $C^{f}$ and can be solved by preconditioned Krylov methods (such as Bi-CGStab or GMRES) with incomplete LU preconditioner [32]. The linear system in step 2 is a little more critical. We remark at first that (28) can be equivalently reformulated as:

$$
\begin{aligned}
& \hat{S} \mathbf{P}^{n+1}=\left(\mathbf{F}_{2}^{n+1}-B^{f} \widetilde{\mathbf{U}}_{0}^{n+1}-\hat{S}_{1} \mathbf{U}_{\Gamma}^{n+1}\right), \\
& \left(\hat{S}_{3}-\hat{S}_{2} \hat{S}^{-1} \hat{S}_{1}\right) \mathbf{U}_{\Gamma}^{n+1}=\mathbf{F}_{3}^{n+1}-C^{s f} \widetilde{\mathbf{U}}_{0}^{n+1}-\hat{S}_{2}(\hat{S})^{-1}\left(\mathbf{F}_{2}^{n+1}-B^{f} \widetilde{\mathbf{U}}_{0}^{n+1}\right) \widetilde{\mathbf{U}}_{0}^{n+1} .
\end{aligned}
$$

We will provide in the next Proposition a sufficient condition that guarantees that matrix $\hat{S}_{3}-\hat{S}_{2} \hat{S}^{-1} \hat{S}_{1}$ is positive definite. This will prove that the existence and uniqueness of $\mathbf{U}_{\Gamma}^{n+1}$. Then from the first equation in (30) we derive that $\mathbf{P}^{n+1}$ exists and is unique too, since $\hat{S}$ is symmetric, negative definite if the inf-sup condition is satisfied (thus $B^{f}$ is a full-rank matrix).

Proposition 3.1 Suppose we are using quasi-uniform conforming meshes whose elements have maximum diameter $h$. We assume that, for a suitable positive constant $C$ (to be introduced later),

$$
h<\frac{2}{C} \frac{\rho^{s}}{\rho^{f}} .
$$


A sufficient condition for matrix $\hat{S}_{3}-\hat{S}_{2} \hat{S}^{-1} \hat{S}_{1}$ to be positive definite is that the time-step $\delta$ t obey the following restriction:

$$
\delta t \leq \delta t_{c r}=\frac{h^{2}}{C_{I}^{2} \gamma^{s f}}\left(-1+\sqrt{\frac{2}{C h} \frac{\rho^{s}}{\rho^{f}}}\right),
$$

where $\gamma^{\text {sf }}$ is the continuity constant of the operator associated to $A^{s f}$ (33) and $C_{I}$ is the constant in the inverse inequality (see (34) below). Then, equation (30) 2 has a unique solution $\mathbf{U}_{\Gamma}^{n+1}$, system (28) is non-singular and the Yosida based semi-implicit algorithm (26)-(27)-(28)-(29) is well defined.

Proof. Since $\forall \boldsymbol{\eta} \in \mathbb{R}^{\mathcal{N}_{s}}$, with $\boldsymbol{\eta} \neq \mathbf{0}$, we have that $-\boldsymbol{\eta}^{T} \hat{S}_{2} \hat{S}^{-1} \hat{S}_{1} \boldsymbol{\eta} \geq 0, \forall t>0$, being $\hat{S}_{2}=\left(\hat{S}_{1}\right)^{T}$, it suffices to prove that (31) and (32) are sufficient conditions for matrix $\hat{S}_{3}$ to be positive definite.

We consider:

$$
\boldsymbol{\eta}^{T} \hat{S}_{3} \boldsymbol{\eta}=\boldsymbol{\eta}^{T}\left(\frac{2}{\delta t} \frac{\rho^{s}}{\rho^{f}} M^{s}+\frac{1}{\delta t} M^{s b}+A^{s}-\delta t C^{s f}\left(M^{f}\right)^{-1} C^{f s}\right) \boldsymbol{\eta},
$$

where matrix $A^{s}$ is defined as follows:

$$
A^{s}=K^{s}+K^{s b}+N^{s b}\left(\mathbf{u}^{*}\right),
$$

with $K_{i, j}^{s b}=\nu \int_{\Omega f, n+1} \nabla \boldsymbol{\phi}_{j}^{b}: \nabla \mathcal{E}_{h} \boldsymbol{\psi}_{i}$ and $N_{i, j}^{s b}\left(\mathbf{u}^{*}\right)=\int_{\Omega^{f, n+1}}\left[\left(\mathbf{u}^{*}-\mathbf{w}_{h}^{n+1}\right) \cdot \nabla\right] \boldsymbol{\phi}_{j}^{b} \cdot \mathcal{E}_{h} \boldsymbol{\psi}_{i}$. Note that the entries of $A^{s}$ are independent of time-step $\delta t$.

Multiplying both members by $\delta t$, we find:

$$
\delta t \boldsymbol{\eta}^{T} \hat{S}_{3} \boldsymbol{\eta}=2 \frac{\rho^{s}}{\rho^{f}}\left\|\boldsymbol{\eta}_{H}\right\|_{L^{2}(\Gamma)}^{2}+\left\|\mathcal{E}_{h} \boldsymbol{\eta}_{H}\right\|_{L^{2}\left(\Omega^{f}\right)}^{2}+\delta t \boldsymbol{\eta}^{T} A^{s} \boldsymbol{\eta}-\delta t^{2} \boldsymbol{\eta}^{T} C^{s f}\left(M^{f}\right)^{-1} C^{f s} \boldsymbol{\eta} .
$$

For geometric conforming meshes $\mathcal{E}_{h} \boldsymbol{\psi}_{i}=\phi_{i}^{b}$, for $i=1, \ldots, \mathcal{N}_{s}$ with $\mathcal{N}_{s}=\mathcal{N}_{v}^{b}$. Then matrix $M^{s b}$ is symmetric and associated to a scalar product: $\boldsymbol{\eta}^{T} M^{s b} \boldsymbol{\eta}=\left\|\mathcal{E}_{h} \boldsymbol{\eta}_{H}\right\|_{L^{2}\left(\Omega^{f}\right)}^{2}$, with $\mathcal{E}_{h} \boldsymbol{\eta}_{H}=\sum_{i=1}^{\mathcal{N}_{s}} \eta_{i} \phi_{i}^{b}$. For matrix $A^{s}$ we have:

$$
\boldsymbol{\eta}^{T} A^{s} \boldsymbol{\eta}=\boldsymbol{\eta}^{T} K^{s} \boldsymbol{\eta}+\boldsymbol{\eta}^{T}\left(K^{s b}+N^{s b}\left(\mathbf{u}^{*}\right)\right) \boldsymbol{\eta} \geq \alpha^{s}\left\|\boldsymbol{\eta}_{H}\right\|_{H^{1}(\Gamma)}^{2}+\alpha^{s b}\left\|\mathcal{E}_{h} \boldsymbol{\eta}_{H}\right\|_{H^{1}\left(\Omega^{f}\right)}^{2},
$$

where $\alpha^{s}$ and $\alpha^{s b}$ are the coercivity constants of the operators associated respectively to $K^{s}$ and $\left(K^{s b}+N^{s b}\left(\mathbf{u}^{*}\right)\right)$.

Set $\mathbf{u}=\left(M^{f}\right)^{-1} C^{f s} \boldsymbol{\eta}$. Then:

$$
\begin{aligned}
\boldsymbol{\eta}^{T} C^{s f} \mathbf{u} & =\boldsymbol{\eta}^{T}\left(\frac{1}{\delta t} M^{s f}+A^{s f}\right) \mathbf{u}=\frac{1}{\delta t} \boldsymbol{\eta}^{T} M^{s f} \mathbf{u}+\boldsymbol{\eta}^{T} A^{s f} \mathbf{u} \\
& \leq \frac{1}{\delta t}\left\|\mathcal{E}_{h} \boldsymbol{\eta}_{H}\right\|_{L^{2}\left(\Omega^{f}\right)}\left\|\mathbf{u}_{h}\right\|_{L^{2}\left(\Omega^{f}\right)}+\gamma^{s f}\left\|\mathcal{E}_{h} \boldsymbol{\eta}_{H}\right\|_{H^{1}\left(\Omega^{f}\right)}\left\|\mathbf{u}_{h}\right\|_{H^{1}\left(\Omega^{f}\right)} \\
& \leq\left(\frac{1}{\delta t}+\frac{C_{I}^{2} \gamma^{s f}}{h^{2}}\right)\left\|\mathcal{E}_{h} \boldsymbol{\eta}_{H}\right\|_{L^{2}\left(\Omega^{f}\right)}\left\|\mathbf{u}_{h}\right\|_{L^{2}\left(\Omega^{f}\right)},
\end{aligned}
$$

where $\gamma^{s f}=\gamma^{s f}\left(\left\|\mathbf{u}^{*}-\mathbf{w}_{h}^{n+1}\right\|\right)$ is the continuity constant of the operator associated to:

$$
A^{s f}=K^{s f}+N^{s f}\left(\mathbf{u}^{*}\right),
$$


with $K_{i, j}^{s f}=\nu \int_{\Omega^{f, n+1}} \nabla \phi_{j}: \nabla \mathcal{E}_{h} \boldsymbol{\psi}_{i}$ and $N^{s f}\left(\mathbf{u}^{*}\right)=\int_{\Omega^{f, n+1}}\left[\left(\mathbf{u}^{*}-\mathbf{w}_{h}^{n+1}\right) \cdot \nabla\right] \boldsymbol{\phi}_{j} \cdot \mathcal{E}_{h} \boldsymbol{\psi}_{i}$, and $C_{I}$ is the constant showing up in the following inverse inequality:

$$
\left\|\mathbf{v}_{h}\right\|_{H^{1}\left(\Omega^{f}\right)} \leq C_{I} h^{-1}\left\|\mathbf{v}_{h}\right\|_{L^{2}\left(\Omega^{f}\right)}, \quad \forall \mathbf{v}_{h} \in V_{h}^{f} .
$$

that holds under the assumption that the triangulation in $\Omega^{f}$ is quasi-uniform (see, e.g., [30]). Since $C^{f s} \boldsymbol{\eta}=M^{f} \mathbf{u}$, it follows that $\boldsymbol{\eta}^{T} C^{s f}=\mathbf{u}^{T} M^{f}$ and so:

$$
\left(\frac{h^{2}+C_{I}^{2} \gamma^{s f} \delta t}{h^{2} \delta t}\right)\left\|\mathcal{E}_{h} \boldsymbol{\eta}_{H}\right\|_{L^{2}\left(\Omega^{f}\right)}\left\|\mathbf{u}_{h}\right\|_{L^{2}\left(\Omega^{f}\right)} \geq \boldsymbol{\eta}^{T} C^{s f} \mathbf{u}=\mathbf{u}^{T} M^{f} \mathbf{u}=\left\|\mathbf{u}_{h}\right\|_{L^{2}\left(\Omega^{f}\right)}^{2} .
$$

Therefore we get $\left\|\mathbf{u}_{h}\right\|_{L^{2}\left(\Omega^{f}\right)} \leq\left(\frac{h^{2}+C_{I}^{2} \gamma^{s f} \delta t}{h^{2} \delta t}\right)\left\|\mathcal{E}_{h} \boldsymbol{\eta}_{H}\right\|_{L^{2}\left(\Omega^{f}\right)}$.

Using Lemma 1 in [7], which states that

$$
\left\|\mathcal{E}_{h} \boldsymbol{\eta}_{H}\right\|_{L^{2}\left(\Omega^{f}\right)}^{2} \leq C h\left\|\boldsymbol{\eta}_{H}\right\|_{L^{2}(\Gamma)}^{2}, \quad \forall \boldsymbol{\eta}_{H} \in \hat{V}_{H}^{s},
$$

where $C>0$ is a mesh-independent constant, we have:

$$
\begin{aligned}
\delta t \boldsymbol{\eta}^{T} \hat{S}_{3} \boldsymbol{\eta} \geq & 2 \frac{\rho^{s}}{\rho^{f}}\left\|\boldsymbol{\eta}_{H}\right\|_{L^{2}(\Gamma)}^{2}+\left\|\mathcal{E}_{h} \boldsymbol{\eta}_{H}\right\|_{L^{2}\left(\Omega^{f}\right)}^{2}+ \\
& \delta t\left(\alpha^{s}\left\|\boldsymbol{\eta}_{H}\right\|_{H^{1}(\Gamma)}^{2}+\alpha^{s b}\left\|\mathcal{E}_{h} \boldsymbol{\eta}_{H}\right\|_{H^{1}\left(\Omega^{f}\right)}^{2}\right)-\delta t \boldsymbol{\eta}^{T}\left(M^{s f}+\delta t A^{s f}\right) \mathbf{u} \\
\geq & 2 \frac{\rho^{s}}{\rho^{f}}\left\|\boldsymbol{\eta}_{H}\right\|_{L^{2}(\Gamma)}^{2}+\left\|\mathcal{E}_{h} \boldsymbol{\eta}_{H}\right\|_{L^{2}\left(\Omega^{f}\right)}^{2}+\delta t\left(\alpha^{s}\left\|\boldsymbol{\eta}_{H}\right\|_{H^{1}(\Gamma)}^{2}+\right. \\
& \left.\alpha^{s b}\left\|\mathcal{E}_{h} \boldsymbol{\eta}_{H}\right\|_{H^{1}\left(\Omega^{f}\right)}^{2}\right)-\delta t\left(1+\delta t \frac{C_{I}^{2} \gamma^{s f}}{h^{2}}\right)\left\|\mathcal{E}_{h} \boldsymbol{\eta}_{H}\right\|_{L^{2}\left(\Omega^{f}\right)}\left\|\mathbf{u}_{h}\right\|_{L^{2}\left(\Omega^{f}\right)} \\
\geq & 2 \frac{\rho^{s}}{\rho^{f}}\left\|\boldsymbol{\eta}_{H}\right\|_{L^{2}(\Gamma)}^{2}+\left\|\mathcal{E}_{h} \boldsymbol{\eta}_{H}\right\|_{L^{2}\left(\Omega^{f}\right)}^{2}+\delta t\left(\alpha^{s}\left\|\boldsymbol{\eta}_{H}\right\|_{H^{1}(\Gamma)}^{2}+\right. \\
& \left.\alpha^{s b}\left\|\mathcal{E}_{h} \boldsymbol{\eta}_{H}\right\|_{H^{1}\left(\Omega^{f}\right)}^{2}\right)-\left(\frac{h^{2}+C_{I}^{2} \gamma^{s f} \delta t}{h^{2}}\right)^{2} C h\left\|\boldsymbol{\eta}_{H}\right\|_{L^{2}(\Gamma)}^{2} \\
\geq & {\left[2 \frac{\rho^{s}}{\rho^{f}}-\frac{C}{h^{3}}\left(h^{2}+C_{I}^{2} \gamma^{s f} \delta t\right)^{2}\right]\left\|\boldsymbol{\eta}_{H}\right\|_{L^{2}(\Gamma)}^{2}+\left\|\mathcal{E}_{h} \boldsymbol{\eta}_{H}\right\|_{L^{2}\left(\Omega^{f}\right)}^{2}+} \\
& \delta t\left(\alpha^{s}\left\|\boldsymbol{\eta}_{H}\right\|_{H^{1}(\Gamma)}^{2}+\alpha^{s b}\left\|\mathcal{E}_{h} \boldsymbol{\eta}_{H}\right\|_{H^{1}\left(\Omega^{f}\right)}^{2}\right) .
\end{aligned}
$$

To simplify the calculations, since the last two terms are positive, we impose the first one to be positive too, that is:

$$
2 \frac{\rho^{s}}{\rho^{f}}-\frac{C}{h^{3}}\left(h^{2}+C_{I}^{2} \gamma^{s f} \delta t\right)^{2}>0 .
$$

This is a more restrictive condition. We calculate the critical time-steps:

$$
\delta t_{c r}^{1,2}=\frac{h^{2}}{C_{I}^{2} \gamma^{s f}}\left(-1 \pm \sqrt{\frac{2}{C h} \frac{\rho^{s}}{\rho^{f}}}\right) .
$$

One of the two critical time-steps must be positive, so we get a restriction on the densities ratio:

$$
\frac{\rho^{s}}{\rho^{f}}>C \frac{h}{2},
$$


which can however be regarded as a restriction on the mesh-size $h$, see (31).

From (36) it follows that, under condition (31), matrix $\hat{S}^{3}$ is positive definite for

$$
\delta t \leq \delta t_{c r}=\frac{h^{2}}{C_{I}^{2} \gamma^{s f}}\left(-1+\sqrt{\frac{2}{C h} \frac{\rho^{s}}{\rho^{f}}}\right) .
$$

This proves our Proposition.

Remark 3.1 In order to simplify the proof of the non-singularity of system (28), we derived two conditions on mesh-size and time-step (respectively (31) and (32)) which are more restrictive than necessary.

Numerical evidence suggests that matrix $\hat{S}_{3}-\hat{S}_{2} \hat{S}^{-1} \hat{S}_{1}$ is positive definite for all the physical and discrete parameters we tested.

Remark 3.2 In view of our previous results, for $\delta t \leq \delta t_{c r}$, matrix

$$
\left[\begin{array}{cc}
\hat{S} & \hat{S}_{1} \\
\hat{S}_{2} & \hat{S}_{3}
\end{array}\right]
$$

is indefinite, however its eigenvalues are real with variable sign. The system (28) has therefore a unique solution and we solve it by a preconditioned Krylov method with incomplete $L U$ preconditioner.

The explicit treatment of the expensive ALE-advection-viscous term is based on the approximation of the domain shape at time $t^{n+1}$ with the domain $\Omega^{n}$ calculated at the previous time-step (Step 0). A standard strongly coupled approach, with $\hat{\mathbf{d}}^{n+1}=\operatorname{Ext}\left(\boldsymbol{\eta}_{\mid \hat{\Gamma}}^{n+1}\right)$ at step 0 , would require to iterate the whole procedure, increasing the overall computational cost. Thanks to the implicit coupling of the pressure (Step 2) we can avoid these iterations without severely affecting the stability.

\subsection{The Yosida scheme vs the algebraic version of the Chorin- Temam method for fluid-structure interaction}

The Yosida scheme is just one out of many algebraic fractional step methods which could be extended to fluid-structure interaction problems.

Another possibility, suggested by the scheme in [7], would be to adapt the algebraic version of the Chorin-Temam method (e.g. [28]) to solve the system (11). In this case matrix $A^{n+1}$ would be approximated by $\bar{A}^{n+1}=\hat{L} \bar{U}$, where

$$
\bar{U}=\left[\begin{array}{ccc}
I & \delta t\left(M^{f}\right)^{-1}\left(B^{f}\right)^{T} & \delta t\left(M^{f}\right)^{-1} C^{f s} \\
0 & I & \hat{S}^{-1} \hat{S}_{1} \\
0 & 0 & I
\end{array}\right]
$$

only the form of the approximate factor $\bar{U}$ changes with respect to the Yosida inexact factors (22). The algebraic Chorin-Temam method for fluid-structure 
interaction shares step (24) and equations $(25)_{1,2}$ with the Yosida based scheme but it replaces equation $(25)_{3}$ with:

$$
\frac{1}{\delta t} M^{f} \mathbf{U}_{0}^{n+1}+\left(B^{f}\right)^{T} \mathbf{P}^{n+1}+C^{f s} \mathbf{U}_{\Gamma}^{n+1}=\frac{1}{\delta t} M^{f} \widetilde{\mathbf{U}}_{0}^{n+1} .
$$

This means that by the simple substitution of equation (29) with equation (37) in Step 3, we obtain a semi-implicit algorithm based on the algebraic version of the Chorin-Temam scheme. This algorithm, its analogies and differences from the algebraic counterpart of the method proposed in [7] will be presented in a future work.

In this report we focus on the Yosida based semi-implicit algorithm (26)(27)-(28)-(29).

\section{Application: blood flow in large arteries}

In this section we illustrate the numerical performances of the coupling scheme proposed in Sec. 3 on a simplified blood flow problem.

\subsection{A generalized string model}

We consider a very simple structure model derived from the equations of linear elasticity for a cylindrical tube of small thickness, under the hypotheses of plane stress and membrane deformations (i.e. negligible elastic bending terms). Given a cylindrical reference surface of radius $R_{0}$, we neglect the longitudinal and angular displacement while the radial displacement $\eta=\eta(z, t)$ satisfies:

$$
\rho^{s} h \frac{\partial^{2} \eta}{\partial t^{2}}-k G h \frac{\partial^{2} \eta}{\partial z^{2}}+\frac{E h}{1-\nu^{2}} \frac{\eta}{R_{0}^{2}}-\gamma \frac{\partial^{3} \eta}{\partial z^{2} \partial t}=f_{\Gamma}(z, t) .
$$

Here $z$ indicates the axial direction, $h$ is the wall thickness, $k$ is the so called Timoshenko shear correction factor, $G, E$ and $\nu$ are respectively the shear modulus, the Young modulus and the Poisson ratio, while $\gamma$ is a viscoelastic parameter. Finally $f_{\Gamma}(z, t)$ is an external forcing term. The term $k G h \frac{\partial^{2} \eta}{\partial z^{2}}$ accounts for shear deformations, while $\gamma \frac{\partial^{3} \eta}{\partial z^{2} \partial t}$ introduces the viscoelastic behavior. This model has been widely used in previous works devoted to blood flows (see, for instance, $[29,26])$.

The conditions $\eta=0$ at $z=0$ and $z=L$, corresponding to clamped wall ends, are not realistic in the blood flow context. Since the structural model is of propagative type, first order absorbing boundary conditions are a better choice:

$$
\frac{\partial \eta}{\partial t}-\sqrt{\frac{k G}{\rho^{s}}} \frac{\partial \eta}{\partial z}=0 \text { at } z=0, \frac{\partial \eta}{\partial t}+\sqrt{\frac{k G}{\rho^{s}}} \frac{\partial \eta}{\partial z}=0 \text { at } z=L .
$$




\subsection{A simplified 2D problem}

We want to simulate the propagation of a pressure pulse coming from the heart in a straight artery of length $L$. Blood can be assumed to behave like a Newtonian fluid in large arteries (those whose diameter is larger than $0.2 \mathrm{~cm}$ ca). To this purpose we adopt a $2 \mathrm{D}$ model obtained by intersecting a portion of blood flow vessel with a plane. The $2 \mathrm{D}$ problem arises from the combination of the $2 \mathrm{D}$ Navier-Stokes equations for the fluid with the generalized string model (38) to describe the motion of the upper and lower boundaries. This $2 \mathrm{D}$ benchmark proposed in [10] maintains the peculiar aspects of the coupled fluid-structure problem, although it is not completely realistic for blood flow problems.

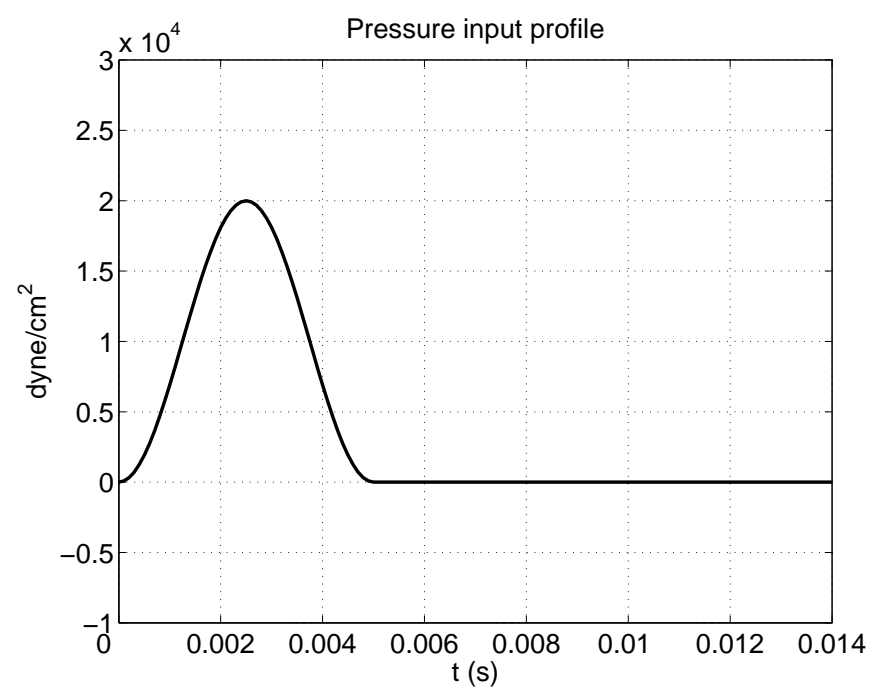

Figure 2: Input profile of the inflow Neumann boundary condition on the normal stress.

\subsection{Numerical results}

The initial domain is a rectangle of height $H=1 \mathrm{~cm}$ and length $L=6 \mathrm{~cm}$, whose upper and lower edges are deformable in the vertical direction.

As in [22], we take the following parameters for the fluid: $\mu=0.035$ poise, $\rho^{f}=1 \mathrm{gr} / \mathrm{cm}^{3}$; and for the structure: $\rho^{s}=1.1 \mathrm{gr} / \mathrm{cm}^{3}, h=0.1 \mathrm{~cm}, E=$ $0.75 \cdot 10^{6}$ dynes $/ \mathrm{cm}^{2}, \nu=0.5, k=1$ and $\gamma=10^{-2}$ dyne $\cdot s$. These parameters have been chosen in the physiological range for a human body.

On the inflow section we impose the following Neumann boundary condition:

$$
\boldsymbol{\sigma}_{i n}^{f}=-\frac{P_{i n}}{2}\left[1-\cos \left(\frac{\pi t}{2.5 \cdot 10^{-3}}\right)\right] \mathbf{n},
$$


while on the outflow section an homogeneous Neumann condition has been imposed. The amplitude $P_{i n}$ of the pressure pulse has been taken equal to $2 \cdot 10^{4}$ dynes $/ \mathrm{cm}^{2}$ and the time duration of the pulse is $5 \mathrm{~ms}$. Fig. 2 shows the input profile $\boldsymbol{\sigma}_{i n}^{f} \cdot \mathbf{n}$. We solve the problem over the time interval [0,0.012] $s$.

We choose a conforming space discretization between fluid and structure: $\left(\mathbb{P}_{1}\right.$ iso $\left.\mathbb{P}_{2}\right)-\mathbb{P}_{1}$ finite elements for the fluid and $\mathbb{P}_{1}$ finite elements for the structure. We have solved the problem with algorithm described in Sec. 3 on the elliptic mesh of $31 \times 21 \mathbb{P}_{1}$ fluid nodes $\left(2501 \mathbb{P}_{1}\right.$ iso $\mathbb{P}_{2}$ nodes $)$ shown in Fig. 3 .

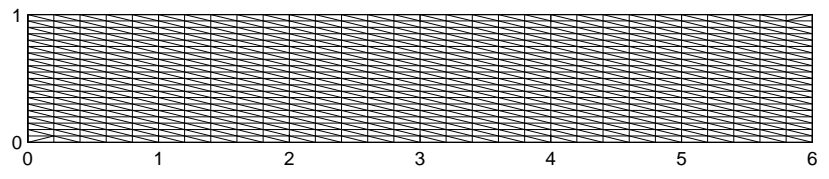

Figure 3: Elliptic mesh used for the simulations.

Fig. 4 shows the fluid pressure contour lines together with the structural deformation at time $t=4,8$ and $12 \mathrm{~ms}$. The solid displacement, definitely nonnegligible, has not been magnified. We see that initial pressure pulse propagates in the artery at a finite speed, although the fluid is modelled as incompressible. The reason of this fact lies in the compliance of the vessel wall.
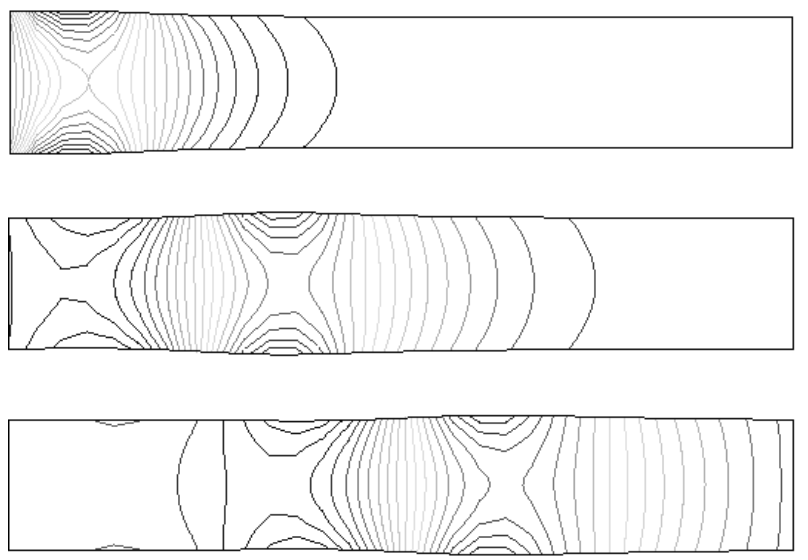

Figure 4: Propagation of the initial pressure pulse, moving from the inflow to the outflow section. Solutions every $4 \mathrm{~ms}$.

We have computed average quantities on each vertical line of the mesh [22], corresponding to the position $z_{i}=i \cdot h$, with $i=0, \ldots, 30$ and $h=0.2 \mathrm{~cm}$. We calculated the diameter of the artery, the average pressure and the flux at each time-step. From Fig. 5, 6 and 7, it is evident that a propagative pulse is associated to all these three quantities. 
In Fig. 5 we report the comparison between the average pressure profiles computed every 2 milliseconds on the same mesh but with two different timesteps $\left(\delta t=10^{-4}\right.$ and $\left.\delta t=10^{-6}\right)$. Fig. 6 shows the same comparison for the flow rate. The choice of discretizing the fluid equations with the implicit Euler scheme and the structure equation with the mid-point rule turns out to be quite dissipative.

The diameter of the artery section along its axis is reported in Fig. 7. In order to understand the influence of spatial discretization on the numerical solution, we compare the solution calculated on the mesh of Fig. 3 with the solution computed on a refined grid. The fine grid has $61 \times 26 \mathbb{P}_{1}$ fluid nodes (6171 $\mathbb{P}_{1}$ iso $\mathbb{P}_{2}$ nodes). In both cases the time-step is $\delta t=10^{-4}$. We notice that the solution for the fine grid is slightly faster than the one computed on the coarse grid, as already pointed out in [22].
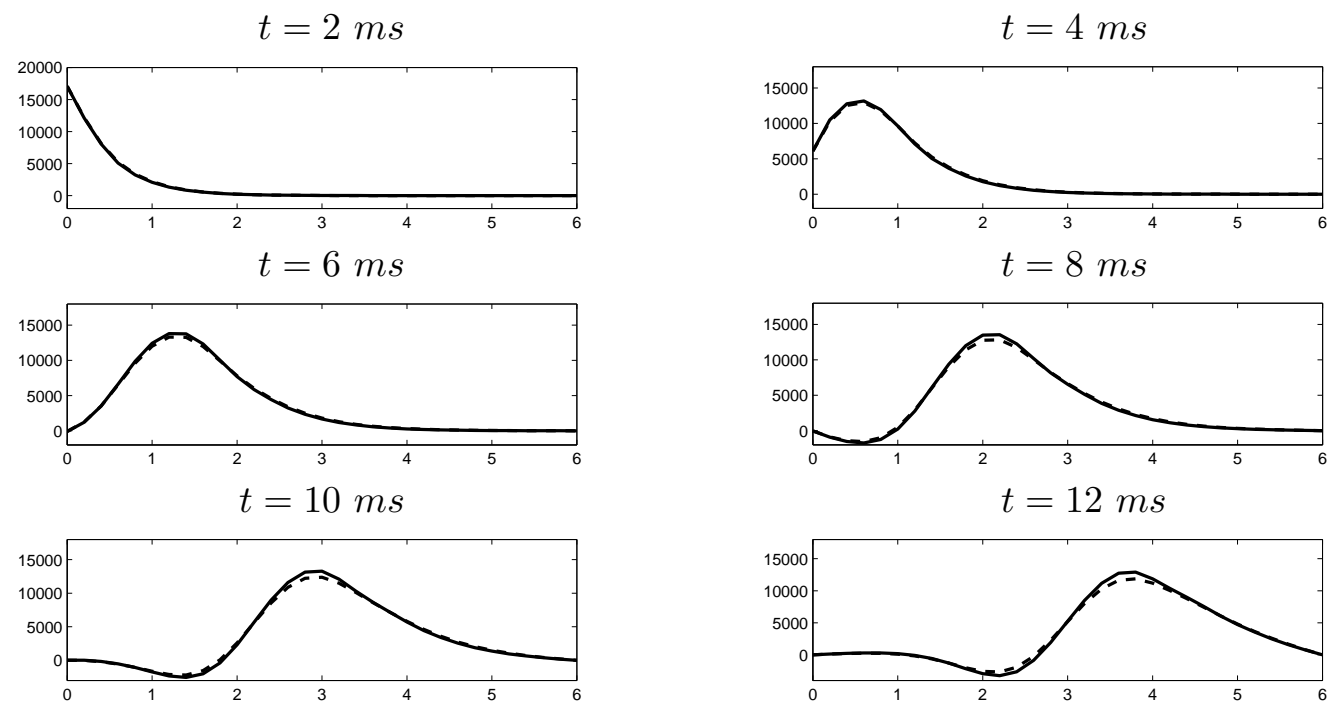

Figure 5: Average pressure profiles at different time levels for $\delta t=10^{-4}$ (dashed line) and for $\delta t=10^{-6}$ (solid line).

\subsection{Accuracy of the algorithm}

We solve the problem with a sequence of decreasing time-steps: $\delta t=4 \cdot 10^{-4}, 2$. $10^{-4}, 10^{-4}, 5 \cdot 10^{-5}, 4 \cdot 10^{-5}$, on the mesh in Fig. 3. We compute the solution of a semi-implicit monolithic algorithm on the same mesh but with a timestep $\delta t=10^{-6}$ : we will address to this solution as the "exact" solution. The semi-implicit monolithic scheme shares the Step 0 with the algorithm of Sec. 3 but solves the system (11) by a global preconditioned GMRES. We compare the solutions computed by the Yosida based method at the different time-steps 
$t=2 \mathrm{~ms}$
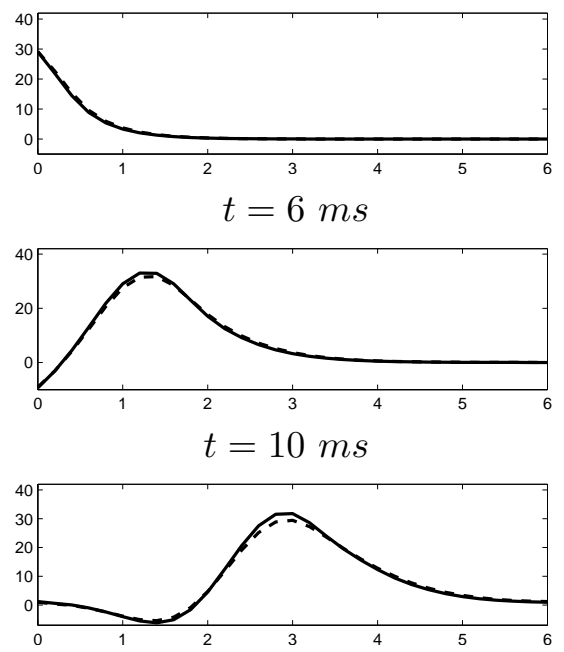

$t=4 \mathrm{~ms}$
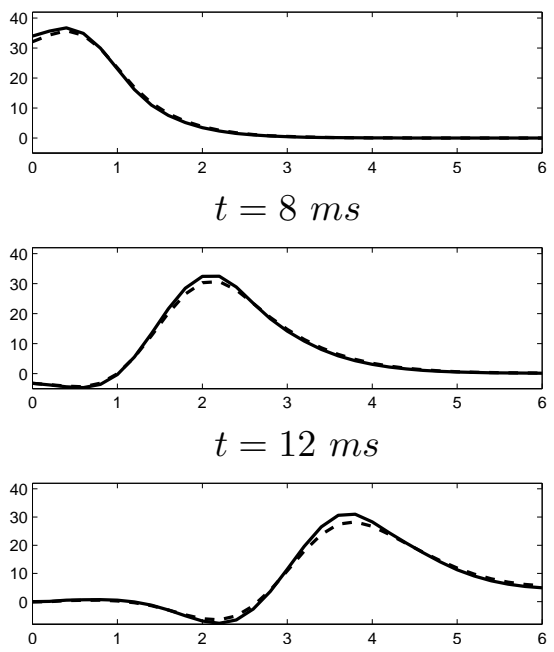

Figure 6: Flow rate profiles at different time levels for $\delta t=10^{-4}$ (dashed line) and for $\delta t=10^{-6}$ (solid line).
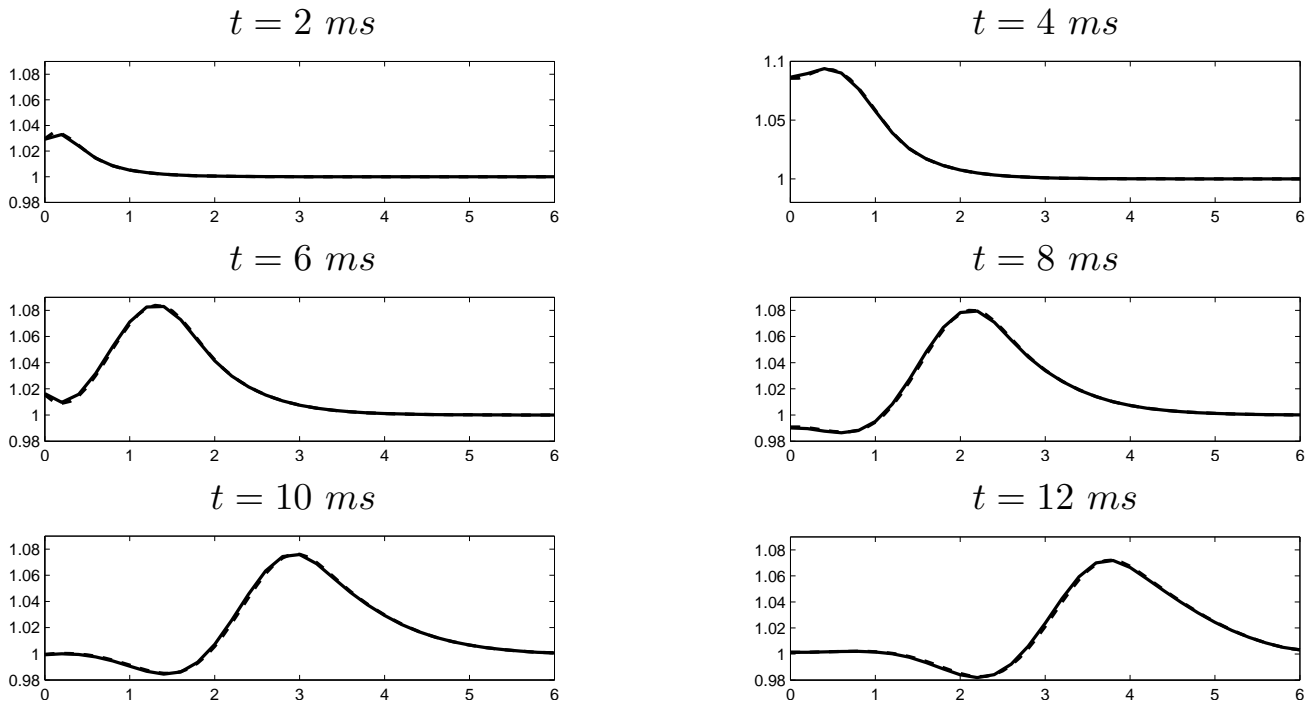

Figure 7: Diameter of the artery section along its axis at different time levels for the coarse mesh (solid line) and for the fine mesh (dashed line).

with the "exact" solution. We repeat the same procedure on the refined grid $61 \times 26$. Fig. 8 shows the error on the fluid velocity, pressure and the structure displacement at time $t=10 \mathrm{~ms}$, both evaluated in the $L^{2}$-norm, for the two meshes. We remark that the "exact" solutions are different for the two grids, so 

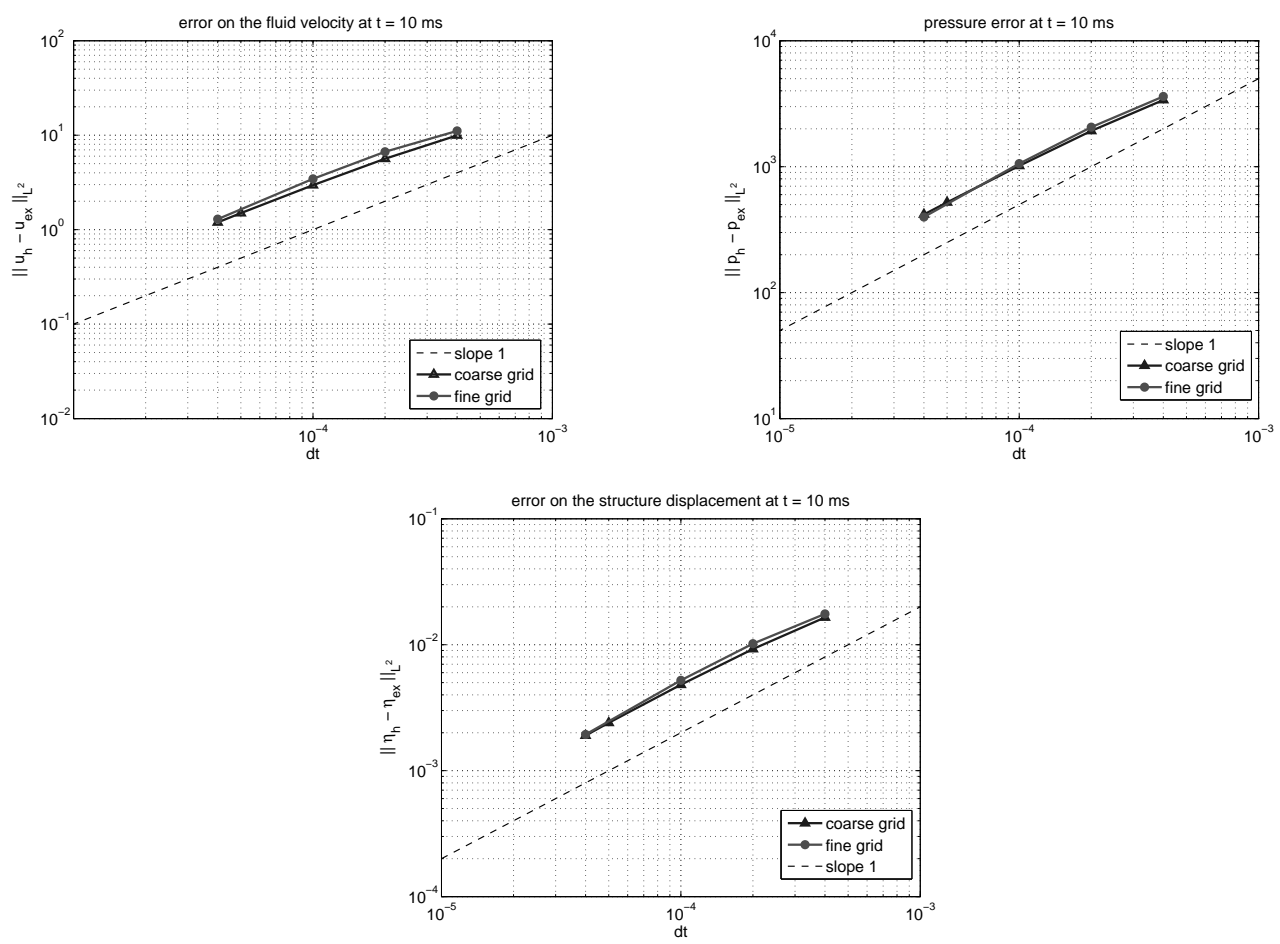

Figure 8: Error on the fluid velocity, pressure and structure displacement in the $L^{2}$-norm at time $t=10 \mathrm{~ms}$ : difference between the solution of the Yosida based algorithm and the "exact" solution.

it can happen that the errors on the fine mesh are bigger than the ones on the coarse grid, as in Fig. 8. As it was expected, since we approximate in time the fluid equations by the backward Euler scheme, we recover a linear convergence (no matter the refinement of the grid).

We check that the solution of a semi-implicit algorithm converges to the solution of an implicit algorithm. We compare the solution of the semi-implicit monolithic method with the solution of the implicit monolithic scheme for $\delta t=$ $10^{-6}$, both on the coarse grid. In Fig. 9 we report the $L^{2}$-error on the fluid velocity and structure displacement. Again we find linear convergence. This means that we can avoid to sub-iterate over the shape domain with important time savings and without compromising the solution accuracy.

In order to evaluate the splitting error introduced by the Yosida method, we compare at the different time-steps the solution of our scheme with the solution of the semi-implicit monolithic method, computed on the same mesh (Fig. 3) and with the same time-step. In Fig. 10 we report the splitting errors for fluid velocity, pressure and structure displacement. The Yosida method introduces an error that behaves like $\delta t^{2}$ for all the three quantities. This means that the 

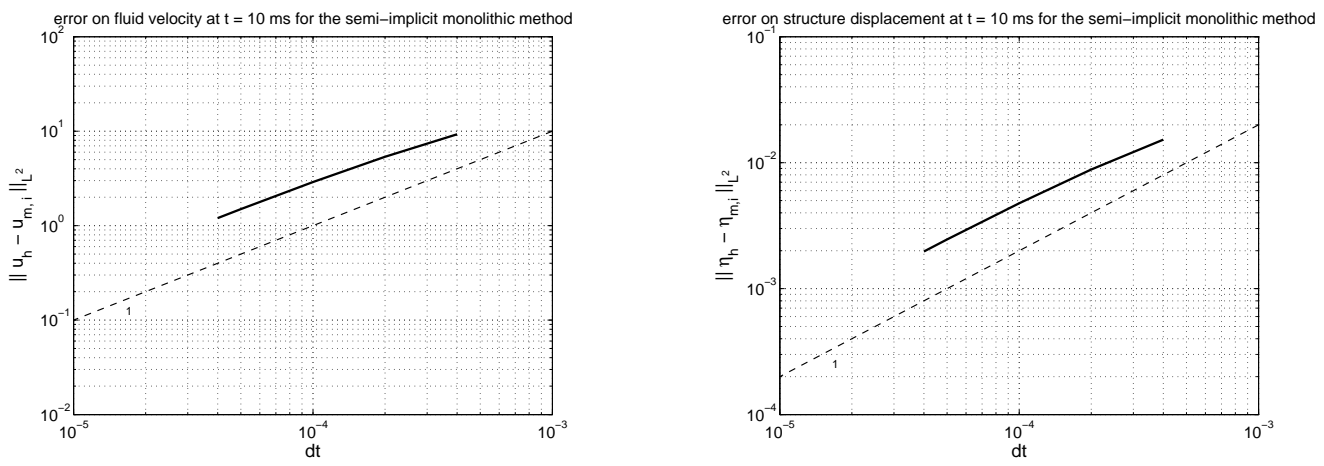

Figure 9: Error on the fluid velocity and structure displacement in the $L^{2}$ norm at time $t=10 \mathrm{~ms}$ : difference between the solution of the semi-implicit monolithic algorithm and the solution of the implicit monolithic algorithm for $\delta t=10^{-6}$.

splitting error is smaller than the time discretization error. Thus, the global error in time for fluid velocity, pressure and structure displacement can be written as the sum of two errors, the first one due to the time discretization scheme and the second one due to the Yosida scheme:

$$
\operatorname{err}(\delta t)=c_{T D} \delta t+c_{Y o s} \delta t^{2},
$$

where $c_{T D}$ and $c_{Y o s}$ are two positive constants independent of $\delta t$. In [14] the same behavior is observed in the case of the Yosida method applied to NavierStokes in a fixed domain. So the empirical formula (39) holds for the Yosida scheme applied to the fluid problem in both moving and fixed domains.

Finally, we can state that the Yosida based semi-implicit coupling scheme converges linearly to the solution of an implicit monolithic scheme with the advantage of great computational cost reduction, due to the explicit treatment of the ALE-convection-viscous term and the use of a fractional step method.

\section{Concluding remarks}

In this paper we have focused on the numerical simulation of fluid-structure interaction problems in the case of an internal flow of incompressible fluid whose density is close to the structure density. Blood flow simulations belong to this category of problems known to be critical for the strong added-mass effect. In such situations fully implicit coupling schemes, preserving the energy balance, are free of instabilities, at the expense of high computational costs, though. Good stability properties are shown by a semi-implicit coupling method introduced in [7]: it remains stable for a wide range of physical and discrete parameters. The basic idea behind that scheme is to couple implicitly the pressure stress 

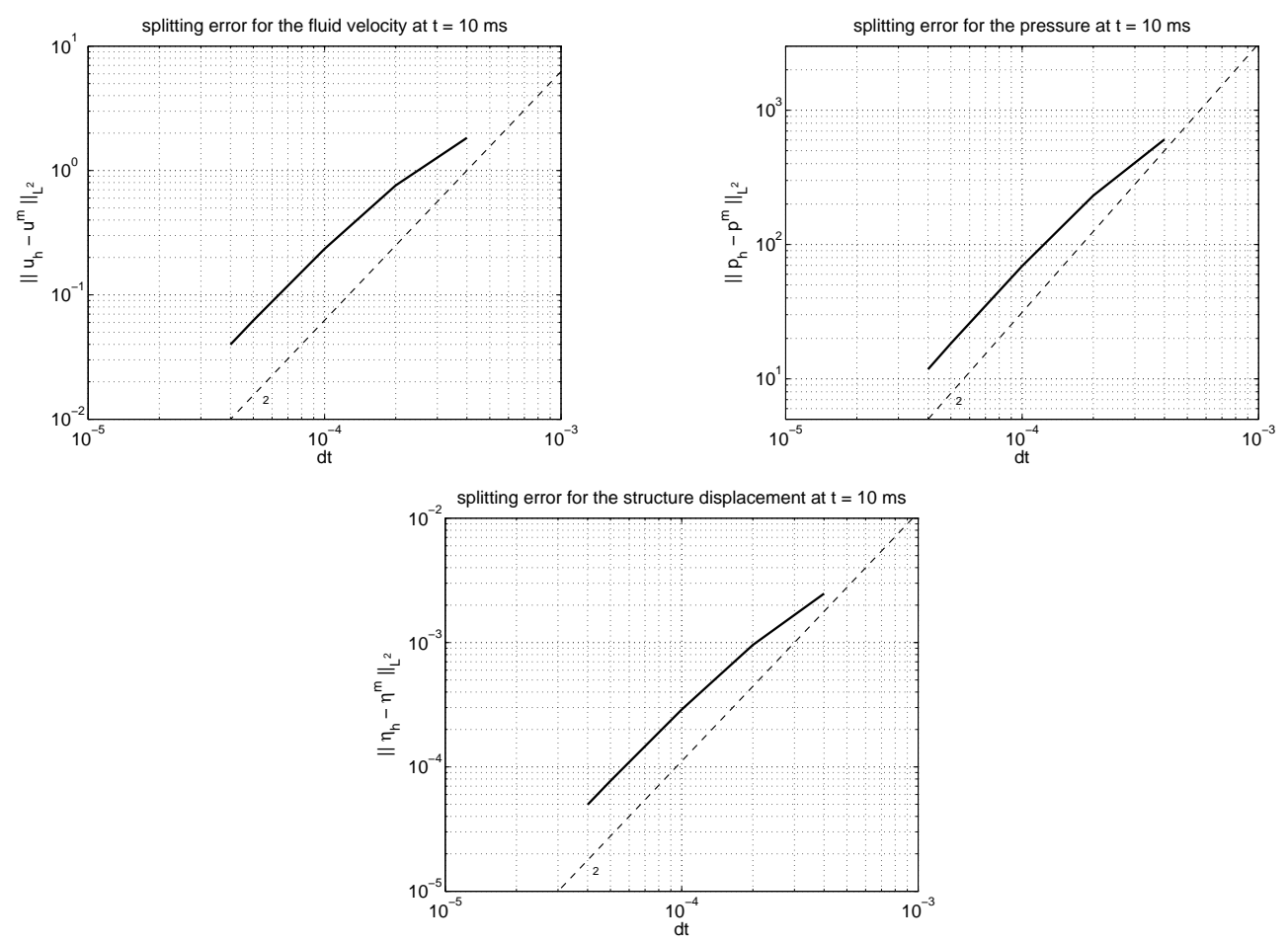

Figure 10: Splitting error on the fluid velocity and structure displacement in the $L^{2}$-norm at time $t=10 \mathrm{~ms}$ : difference between the solution of the Yosida based algorithm and the monolithic solution computed with the same time-step.

to the structure with the double advantage of ensuring stability and reducing computational costs, since the remaining fluid expensive terms are explicitly treated. In [7] they perform the implicit-explicit splitting using a Chorin-Temam projection scheme for the fluid.

In this report we propose an algebraic fractional-step method to split the procedure in explicit and implicit steps. Unlike differential splitting, algebraic fractional-step schemes need no auxiliary boundary conditions for the differential sub-problems in which the original problem is divided. The importance of this feature is even greater for fluid-structure interaction problems, in which the accuracy of the transmission conditions plays a key role.

In our specific example we used the Yosida method to solve a simplified 2D problem simulating the propagation of a pressure pulse in a straight pipe.

There exist many possible ways of extending the work here presented. First of all, we shall evaluate how the added-mass effect affects the Yosida based algorithm. We may apply our method to three-dimensional problems (3D model for the fluid with 2D or 3D model for the structure). More realistic applications would also allow us to compute the computational costs reduction of our scheme 
when compared to other algorithms. We shall also investigate the applicability of other algebraic fractional-step methods, like Yosida scheme modifications called Yosida3 [33] and Yosida4 [14].

\section{Acknowledgements}

We are grateful to Dr. Santiago Badia for several suggestions and insights and to Prof. Fausto Saleri for his help on the implementation. Acknowledgments for the support provided through the Swiss National Science Foundation under grant $200021-109378 / 1$. This work has also been supported by Italian MIUR Cofin2005 "Numerical modelling in fluid dynamics with application to the cardiovascular system and environment".

\section{References}

[1] F. Brezzi and M. Fortin, Mixed and Hybrid Finite Element Methods, Springer Series in Computational Mathematics, 15 (Springer-Verlag, 1991).

[2] P. Causin, J.-F. Gerbeau and F. Nobile, Added-mass effect in the design of partitioned algorithm for fluid-structre interaction, Comp. Meth. Appl. Mech. Engrg. 194 (2005) 4506-4527.

[3] M. Cervera, R. Codina and M. Galindo, On the computational efficiency and implementation of block-iterative algorithms for nonlinear coupled problems, Engrg. Comput. 13 (1996) 4-30.

[4] A. J. Chorin, Numerical solutions of the Navier-Stokes equations, Math. Comput. 22 (1968) 745-762.

[5] S. Deparis, M. Discacciati, G. Fourestey and A. Quarteroni, Heterogeneous domain decomposition methods for fluid-structure interaction problems, Technical report, EPFL-IACS report 08.2005 (2005).

[6] S. Deparis, M. Discacciati and A. Quarteroni, A domain decomposition framework for fluid-struture interation problems, In Proceedings of the Third International Conference on Computational Fluid Dynamics (ICCFD3) (2004).

[7] M. A. Fernández, J.-F. Gerbeau and C. Grandmont, A projection algorithm for fluid-structure interaction problems with strong added-mass effect, $C$. R. Acad. Sci. Paris. 342 (2006) 279-284.

[8] M.A. Fernández and M. Moubachir, An exact block-Newton algorithm for solving fluid-structure interaction problems, C. R. Math. Acad. Sci. Paris 336 (2003) 681-686. 
[9] M.A. Fernández and M. Moubachir, A Newton method using exact Jacobians for solving fluid-Structure coupling, Comp. \& Struct. 83 (2005) $127-142$.

[10] L. Formaggia, J.-F. Gerbeau, F. Nobile and A. Quarteroni, On the coupling of 3D and 1D Navier-Stokes equations for flow problems in compliant vessels, Comp. Meth. Appl. Mech. Engrg. 191 (2001) 561-582.

[11] J.-F. Gerbeau and M. Vidrascu, A quasi-Newton algorithm based on a reduced model for fluid-structure interaction problems in blood flows, Math. Model. Num. Anal. 37 (2003) 631-648.

[12] J.-F. Gerbeau, M. Vidrascu and P.Frey, Fluid-structure interaction in blood flows on geometries based on medical imaging, Comp. \& Struct. 83 (2005) $155-165$.

[13] P. Gervasio, Fractional step methods for spectral approximation of advection-diffsion equations, Math. Models Methods Appl. Sci. 6 (1996) 1027-1050.

[14] P. Gervasio, F. Saleri and A. Veneziani, Algebraic fractional step schemes with spectral methods for the incompressible Navier-Stokes, J. Comp. Phys 214 (2006) 347-365.

[15] C. Grandmont, V. Guimet and Y. Maday, Numerical analysis of some decoupling techniques for the approximation of the unsteady fluid structure interaction, Math. Models Methods Appl. Sci. 11 (2001) 1349-1377.

[16] M. Heil, An efficient solver to the fully coupled solution of largedisplacement fluid-structure interaction problems, Comput. Meth. Appl. Mech. Engrg. 193 (2004) 1-23.

[17] P. Le Tallec and J. Mouro, Fluid-structure interaction with large structural displacements, Comput. Meth. Appl. Mech. Engrg. 190 (2001) 3039-3067.

[18] H.G. Matthies and J. Steindorf. Partitioned but strongly coupled iteration schemes for nonlinear fluid-structure interaction, Comp. \& Struct. 80 (2002) 1991-1999.

[19] H.G. Matthies and J. Steindorf, Partitioned strong coupling algorithms for fluid-structure interaction, Comp. E Struct. 81 (2003) 805-812.

[20] D.P. Mok and W.A. Wall, Patitioned analysis schemes for the transient interaction of incopressible flows and nonlinear flexible strucutres, In K. Schweizerhof, W.A. Wall, K.U. Bletzinger, editor, Trends in Computational Structural Mechanics (Barcelona CIMNE, 2001). 
[21] D.P. Mok, W.A. Wall and E.Ramm, Accelerated iterative substructuring schemes for instationary fluid-structure interaction, In K.J. Bathe, editor, Computational Fluid and Solid Mechanics (Elsevier, 2001).

[22] F. Nobile, Numerical Approximation of Fluid-Structure Interaction Problems with Application to Haemodynamics, PhD Thesis EPFL Switzerland (2001).

[23] B. Perot, An analysis of the fractional step method, J. Comp. Phys. 108 (1993) 51-58.

[24] S. Piperno, Explicit/implicit fluid/structure staggered procedures with a structural predictor and fluid subcycling for $2 \mathrm{D}$ inviscid aeroelastic simulations, Internat. J. Numer. Methods Fluids 25 (1997) 1207-1226.

[25] S. Piperno, C. Farhat and B. Larrouturou, Patitioned procedures for the transient solution of coupled aeroelastic problems. Part I: Model problem, theory and two-dimesional application, Comp. Meth. Appl. Mech. Engrg. 124 (1995) 79-112.

[26] A. Quarteroni and L. Formaggia, Mathematical modelling and numerical simulation of the cardiovascular system, Chapter in Modelling of Living Systems, Handbook of Numerical Analysis Series, P.G. Ciarlet et J.L. Lions Eds. (Elsevier, 2004).

[27] A. Quarteroni, F. Saleri and A. Veneziani, Analysis of the Yosida method for the incompressible Navier-Stokes equations, J. Math. Pures Appl. 78 (1999) 473-503.

[28] A. Quarteroni, F. Saleri and A. Veneziani, Factorization methods for the numerical approximation of Navier-Stokes equations, Comput. Meth. Appl. Mech. Engrg. 188 (2000) 505-526.

[29] A. Quarteroni, M. Tuveri and A. Veneziani, Computational vascular fluid dynamics: Probelms, models and methods, Comp. Vis. Science 2 (2000) 163-197.

[30] A. Quarteroni and A. Valli, Numerical Approximation of Partial Differential Equations (Springer-Verlag, 1994).

[31] S. Rugonyi and K.J. Bathe, On Finite Element analysis of fluid flows coupled with structural interaction. CMES - Comp. Modeling Eng. Sci. 2 (2001) 195-212.

[32] Y. Saad, Iterative Methods for Sparse Linear Systems, Society for Industrial and Applied Mathematics, Philadelphia, PA, second edition (2003). 
[33] F. Saleri and A. Veneziani, Pressure-correction algebraic splitting methods for the incompressible equations, SIAM Journal on Numerical Analysis 43 (2005) 174-194.

[34] R. Temam, Sur l'approximation de la solution des équations de NavierStokes par la méthod de pas fractionaires (II), Arch. Rat. Mech. Anal. 33 (1969) 377-385.

[35] T.E. Tezduyar, Finite Element methods for fluid dynamics with moving boundaries and interfaces, Arch. Comput. Methods Engrg. 8 (2001) 83130.

[36] R. Torii, M. Oshima, T. Kobayashi, K. Takagi and T.E. Tezduyar, Computer modeling of cardiovascular fluid-structure interactions with the Deforming-Spatial-Domain/Stabilized Space-Time formulation, Comput. Meth. Appl. Mech. Engrg. 195 (2006) 1885-1895. 